\title{
Quasicubic model for metal halide perovskite nanocrystals
}

Cite as: J. Chem. Phys. 151, 234106 (2019); https://doi.org/10.1063/1.5127528

Submitted: 11 September 2019 . Accepted: 12 November 2019 . Published Online: 17 December 2019

Peter C. Sercel (D), John L. Lyons (D), Noam Bernstein, and Alexander L. Efros (D)

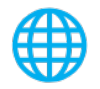

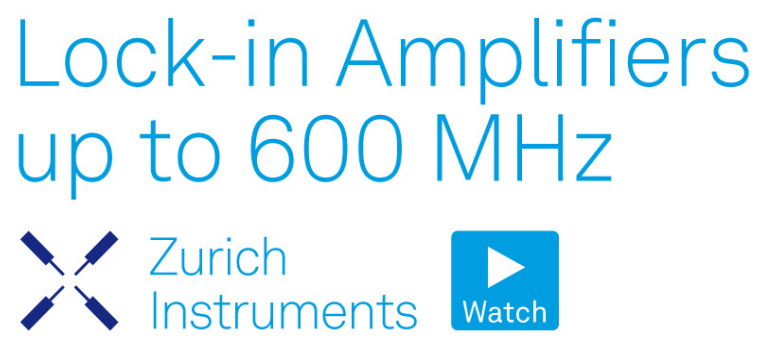

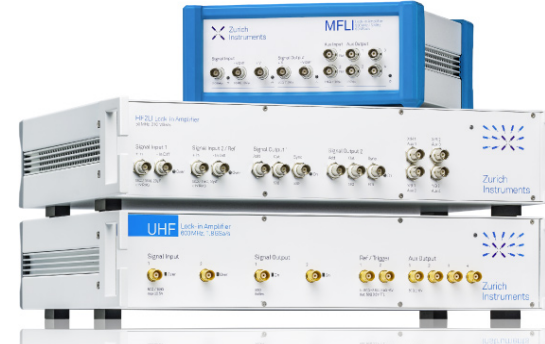

J. Chem. Phys. 151, 234106 (2019); https://doi.org/10.1063/1.5127528

151, 234106 


\title{
Quasicubic model for metal halide perovskite nanocrystals
}

\author{
Cite as: J. Chem. Phys. 151, 234106 (2019); doi: 10.1063/1.5127528 \\ Submitted: 11 September 2019 • Accepted: 12 November 2019 • \\ Published Online: 17 December 2019
}

\author{
Peter C. Sercel, ${ }^{1, a)}$ (D) John L. Lyons, ${ }^{2}$ (D) Noam Bernstein, ${ }^{2}$ and Alexander L. Efros ${ }^{2}$
}

\begin{abstract}
AFFILIATIONS
${ }^{1}$ Department of Applied Physics and Materials Science, California Institute of Technology, Pasadena, California 91125, USA

${ }^{2}$ Center for Computational Materials Science, U.S. Naval Research Laboratory, Washington, DC 20375, USA
\end{abstract}

Note: This paper is part of the JCP Special Topic on Colloidal Quantum Dots.

a) Also at: Center for Hybrid Organic Inorganic Semiconductors for Energy, 15013 Denver West Parkway, Golden,

CO 80401, USA. Electronic mail: psercel@caltech.edu.

\begin{abstract}
We present an analysis of quantum confinement of carriers and excitons, and exciton fine structure, in metal halide perovskite (MHP) nanocrystals (NCs). Starting with coupled-band $\boldsymbol{k} \cdot \boldsymbol{P}$ theory, we derive a nonparabolic effective mass model for the exciton energies in MHP $\mathrm{NCs}$ valid for the full size range from the strong to the weak confinement limits. We illustrate the application of the model to CsPbBr 3 NCs and compare the theory against published absorption data, finding excellent agreement. We then apply the theory of electron-hole exchange, including both short- and long-range exchange interactions, to develop a model for the exciton fine structure. We develop an analytical quasicubic model for the effect of tetragonal and orthorhombic lattice distortions on the exchange-related exciton fine structure in $\mathrm{CsPBr}_{3}$, as well as some hybrid organic MHPs of recent interest, including formamidinium lead bromide $\left(\mathrm{FAPbBr}_{3}\right)$ and methylammonium lead iodide $\left(\mathrm{MAPbI}_{3}\right)$. Testing the predictions of the quasicubic model using hybrid density functional theory (DFT) calculations, we find qualitative agreement in tetragonal MHPs but significant disagreement in the orthorhombic modifications. Moreover, the quasicubic model fails to correctly describe the exciton oscillator strength and with it the long-range exchange corrections in these systems. Introducing the effect of NC shape anisotropy and possible Rashba terms into the model, we illustrate the calculation of the exciton fine structure in $\mathrm{CsPbBr}{ }_{3} \mathrm{NCs}$ based on the results of the DFT calculations and examine the effect of Rashba terms and shape anisotropy on the calculated fine structure.
\end{abstract}

Published under license by AIP Publishing. https://doi.org/10.1063/1.5127528

\section{INTRODUCTION}

Colloidal metal halide perovskite (MHP) nanocrystals (NCs) developed over the last few years exhibit unprecedented, technologically interesting properties. Cesium lead halide $\left.(\mathrm{CsPbX})_{3}\right) \mathrm{NCs}$ have been demonstrated to show bright emission covering the full visible spectral range by virtue of compositional variation and quantum size effects, with $90 \%+$ quantum efficiencies without surface passivation. ${ }^{1}$ The exciton radiative emission in these NCs at a low temperature is very fast, with measured radiative decay rates in the sub-nanosecond range, ${ }^{2} 2-3$ orders of magnitude faster than in other common semiconductor NCs (e.g., $\mathrm{CdSe}^{3}$ or $\mathrm{InP}^{4}$ ). Moreover, the lifetime increases with increasing temperature; ${ }^{5}$ at room temperature, they emit with nanosecond-scale lifetimes, ${ }^{1}$ more than an order of magnitude faster than other semiconductor NCs (e.g., CdSe and InP NCs) that have been optimized for over two decades.

The phenomenology described above does not seem to be consistent with the standard model of exciton fine structure in NCs that worked out over the last 25 years, where, owing to the exchange splitting of the exciton, it is expected that there is always a dark, or dipole inactive, exciton ground state. ${ }^{6,7}$ In the MHPs, owing to the two-fold degeneracy of the conduction and valence bands, each of which can be described as corresponding to angular momentum $1 / 2$ states in the cubic phase, the exchange interaction is expected to split the ground exciton into a dipole-active (bright) triplet state with total angular momentum 1 and a lower energy, dipole-inactive (dark) singlet with total angular momentum 0 . Such a fine structure 
was considered first in $\mathrm{CuCl}^{8}$ Since the exciton would be expected to cool into the lowest state at low temperature, the lifetime should increase as the temperature is reduced. This discrepancy has spurred a series of experimental investigations directed at the detection of the ground dark exciton conducted on perovskite NC ensembles ${ }^{9-11}$ and in single NCs. ${ }^{12-14}$ Indirect evidence for a dark ground-state exciton has been reported based on three-level modeling of the temperature dependence of the photoluminescence decays in conjunction with magnetic brightening either with external fields ${ }^{9,10}$ or via magnetic impurities, ${ }^{11}$ and direct observation of the dark ground exciton state via magnetic activation has been reported in single formamidinium lead bromide $\left(\mathrm{FAPbBr}_{3}\right) \mathrm{NCs}^{15}$ However, similar efforts to directly detect a dark ground exciton in single $\mathrm{Cs} \mathrm{PbBr}_{3} \mathrm{NCs}$ through magnetic brightening have not been successful. ${ }^{12,13}$ The failure to directly detect the expected dark ground exciton state in $\mathrm{CsPbX}_{3}$ $(\mathrm{X}=\mathrm{Cl}, \mathrm{Br}, \mathrm{I}) \mathrm{NCs}$, in conjunction with the unusual temperature dependence of the radiative lifetime in these systems, led to the proposal that the fine structure in $\mathrm{CsPbX}_{3} \mathrm{NCs}$ is determined not by electron-hole exchange but by the Rashba effect, leading to the prediction that the ground exciton state in $\mathrm{CsPbX}_{3} \mathrm{NCs}$ is dipole active. $^{16}$

Apart from the question of the order of levels of the bright vs dark exciton states, there remains the question of what mechanismshort-range (SR) vs long-range exchange, intrinsic crystal field and/or shape anisotropy, or the Rashba effect-determines the order of levels amongst the optically active triplet exciton states in NCs of $\mathrm{CsPbX}_{3}$ and other MHPs, and the magnitude of their splitting. Low temperature investigations of single $\mathrm{CsPbX}_{3} \mathrm{NCs}$ show either a two- or a three-line structure with millielectronvolt-scale splittings (with no observation of lower lying dark exciton states). ${ }^{12-14,16}$ Three-line spectra observed by several groups are associated with emission from three bright excitons, consistent with orthorhombic symmetry. ${ }^{2,12,14,16} \mathrm{CsPbBr}_{3} \mathrm{NCs}$ of apparent tetragonal symmetry consistently show fine structure comprising a low lying linearly polarized line and an upper doublet, which is split in a magnetic field. ${ }^{12}$ The observed level order of a singlet below a doublet has been variously ascribed to being due to intrinsic crystal field splitting, ${ }^{12,14}$ splitting due to shape anisotropy, ${ }^{17-19}$ or the Rashba effect. ${ }^{19}$ In the case of models involving intrinsic crystal field splitting, the magnitude and sign of the crystal field have been inferred from the level structure observed in single NC measurements assuming no other effects such as shape anisotropy; the resulting values estimated from $\mathrm{CsPbBr}_{3} \mathrm{NCs}^{12,14}$ have the opposite sign from the value calculated for tetragonal $\mathrm{CsPBr}_{3}$ using density functional theory (DFT). ${ }^{19}$ Another issue is that the magnitude of the measured bright exciton splitting, that is, the splitting within the bright triplet manifold, which is on the order of $1 \mathrm{meV}$ in $\mathrm{CsPbBr}_{3} \mathrm{NCs}^{2,12,14}$ is larger than the full singlet-triplet splitting expected due to the short-range (SR) exchange interaction splitting calculated using density functional theory (DFT). ${ }^{16,19}$ This has led to several different proposals to explain the magnitude of the bright triplet level spacing in MHP NCs: that it is due to the confinement enhancement of the shortrange exchange interaction; ${ }^{14}$ that the contribution of long-range exchange exceeds that of the short-range contribution; ${ }^{15,17-19}$ or that the Rashba effect ${ }^{16,19}$ determines the bright triplet level spacing in MHP NCs. A quantitative understanding of these various contributions to the exciton fine structure is critical if experimental measurements are to be tied to a microscopic theory of the exciton fine structure. For example, a quantitative exchange model is required to determine whether measurements of the temperature dependence of ensemble photoluminescence, which have been interpreted in conjunction with phenomenological three-level system modeling, ${ }^{10,11}$ can be explained solely within an exciton fine structure model.

Complicating the analysis of the experimental data pertaining to exciton fine structure in MHP NCs is the fact that experimental values for key materials parameters needed to build quantitative models are often unavailable. Indeed, effective masses and dielectric constants, which determine the exciton radius and the NC-size dependence of the exciton transition energies in NCs, have commonly been taken from density functional theory (DFT) calculations in order to model size-dependent exciton structure. ${ }^{1,16,17}$ Taking $\mathrm{CsPbX}_{3}$ as an example, however, the reported effective mass values based on $\mathrm{DFT}^{1,16}$ are significantly different than the recent experimentally measured values in bulk $\mathrm{CsPbX}_{3}{ }^{20}$. Additionally, due to the absence of direct experimental values, the exchange constants have so far been estimated from $\mathrm{DFT}^{16,19}$ for the short-range exchange, and for the long-range exchange, the constant has been estimated from effective masses obtained either from DFT calculations ${ }^{15,17}$ or experimental measurement. ${ }^{18,19}$ In the case of $\mathrm{CsPbBr}_{3}$, the resulting estimates of the LR exchange parameter $\hbar \omega_{L T}$ range from $3.5 \mathrm{meV}^{19}$ to $6.3 \mathrm{meV},{ }^{17}$ while a recent direct measurement gives $5.4 \mathrm{meV}$.

These uncertainties outlined above underscore the need for a comprehensive analysis of the size-dependent quantum confinement energy and exciton fine structure in MHPs. To this end, in this paper, we develop a quasicubic (QC) model for excitons in MHP NCs. In a quasicubic model, we assume cubic symmetry to start with and consider departures from cubic to tetragonal or orthorhombic symmetry (either associated with a lattice distortion or a shape asymmetry) via perturbation theory. We begin with a coupled-band $\boldsymbol{k} \cdot \boldsymbol{P}$ model for the carrier confinement energies derived by Kang and Wise ${ }^{22}$ for spherical NCs, and from it, we derive an isotropic, nonparabolic effective mass model which can be applied to carriers and excitons in MHP NCs. We apply the model to $\mathrm{CsPbBr}_{3} \mathrm{NCs}$ whose size is comparable to or a few times larger than the exciton Bohr radius-that is, in an intermediate confinement regime where correlated electron-hole motion is important-as distinct from the usual strong confinement approximation where NC size is much less than the exciton radius. To establish the validity of the model, we test the coupled-band theory and the effective nonparabolic mass model against absorption data measured in $\mathrm{CsPbB}_{3}$ NCs reported by Brennan et al. ${ }^{23}$ Using parameters derived from the measured bulk effective mass data of Ref. 20, we find excellent agreement with the Brennan NC data. Next, we develop a quasicubic model for the effect of tetragonal and orthorhombic lattice distortions and apply this model to calculate the exchange-related exciton fine structure in cube-shaped $\mathrm{NCs}$ of $\mathrm{CsPbBr}_{3}$ of tetragonal and orthorhombic lattice symmetry. We use the model to predict the exciton bright triplet level order for several hybrid MHPs of interest and compare the results against DFT calculations. Finally, we introduce the effect of NC shape anisotropy and possible Rashba terms into the model. We conclude by calculating the fine structure of tetragonal $\mathrm{CsPBr}_{3} \mathrm{NCs}$, with and without Rashba terms, and discussing the results in the context of available experimental data. 


\section{QUANTUM CONFINED LEVELS IN MHP NANOCRYSTALS}

We begin consideration of the quantum-confined carrier states in metal halide nanocrystals by noting that both the conduction and valence band-edge states in the cubic phase perovskites can be characterized by angular momentum $J=1 / 2{ }^{24}$ As such, the conduction and valence bands are both simple bands without the orbital degeneracy that complicates analysis, for example, of the valence bands in the more familiar III-V and II-VI semiconductors. The simplest model for the quantum confined levels in MHP nanocrystals can therefore reasonably be based on a simple single-band effective mass approximation.

In this approximation, the wavefunction of the ground quantum size level in a cube-shaped NC with edge length $L$ is given by

$$
\psi_{\mathrm{gr}}(x, y, z)=(2 / L)^{3 / 2} \cos (\pi x / L) \cos (\pi y / L) \cos (\pi z / L) \text {. }
$$

Assuming parabolic band dispersion, we compute the ground state confinement energy for this state as

$$
\mathcal{E}_{s}=\frac{\hbar^{2}}{2 m^{*}} 3\left(\frac{\pi}{L}\right)^{2}
$$

where $m^{*}$ is the effective mass of the carrier. We note that exactly the same ground state energy is obtained for a spherical NC with radius $a_{\text {eff }}=L / \sqrt{3}$, which has wavefunction in spherical coordinates of the form

$$
\psi_{\mathrm{gr}}(\boldsymbol{r})=\sqrt{\frac{2 \pi^{2}}{a_{e f f}^{3}}} j_{0}\left(\pi r / a_{e f f}\right) Y_{0}^{0}(\theta, \phi),
$$

where $Y_{0}^{0}(\theta, \phi)$ is the spherical harmonic for the state with angular momentum $l=0$ and angular momentum projection $m=0$ and $j_{l}$ is the spherical Bessel function with index $l=0$. We will therefore refer to the state described by Eq. (1) as $s$-like. We give the corresponding expressions for the excited $p$-like state with its nodal plane perpendicular to $z$,

$$
\begin{aligned}
\psi_{p_{z}}(x, y, z) & =(2 / L)^{3 / 2} \cos (\pi x / L) \cos (\pi y / L) \sin (2 \pi z / L), \\
\mathcal{E}_{p} & =\frac{\hbar^{2}}{2 m^{*}} 6\left(\frac{\pi}{L}\right)^{2} \\
\psi_{p_{z}}(\boldsymbol{r}) & =\sqrt{\frac{2}{a_{e f f}^{3}}} \frac{j_{1}\left(\alpha_{1,1} r / a_{e f f}\right)}{j_{2}\left(\alpha_{1,1}\right)} Y_{1}^{0}(\theta, \phi), \mathcal{E}_{p}=\frac{\hbar^{2}}{2 m^{*}}\left(\frac{\alpha_{1,1}}{a_{e f f}}\right)^{2} .
\end{aligned}
$$

The top line in the equation above gives the wavefunction and energy for a cube-shaped NC, while the lower line shows the expressions for the corresponding state in a spherical NC. In the last line, the term $\alpha_{1,1} \approx 4.493$ is the first root of the spherical Bessel function $j_{1}(x)$. Using the effective radius $a_{\text {eff }}=L / \sqrt{3}$ in the expression for the spherical NC gives a confinement energy within $2.2 \%$ of the result calculated for the cube-shaped NC.

While the parabolic, simple band approach above is certainly valid for sufficiently large NCs, growth and characterization of $\mathrm{CsPBr}_{3} \mathrm{NCs}$ with sizes down to edge lengths of $\sim 3 \mathrm{~nm}$, comparable to the typical exciton radius in these materials, have recently been reported. ${ }^{23}$ For such small NCs, the confinement energies are appreciable and we expect band nonparabolicity and band coupling effects to become important. A model for treating this problem for spherical NCs with isotropic bands, which utilizes the fact that quantum size levels in a spherical NC are eigenstates of total angular momentum, was developed by Kang and Wise ${ }^{22}$. Of course a complication in applying this model to MHP nanocrystals is that the fact that MHP nanocrystals have a nanocube morphology with cubic or nearly cubic shape. ${ }^{1,25}$ The difference between a cubic shape and a spherical shape can be described in terms of a perturbation; writing the perturbation in terms of a spherical harmonic expansion, the lowest order terms in the perturbation potential have angular momentum $L=4$. These terms can have no effect in first order on the lowest energy quantum size levels and only a weak effect in second order since the coupling is via highly excited states. ${ }^{26}$ It is therefore a good approximation to model cube-shaped MHP nanocrystals as spherical with an effective radius $a_{\text {eff }}=L / \sqrt{3}$. As noted above, this correspondence is exact for the ground state and within $\sim 2 \%$ for the excited $p$ states within the simple effective mass approximation.

The energy of quantum confined levels is determined by the boundary conditions. Here, we assume impenetrable boundary conditions. For the lowest quantum confined levels, which have total angular momentum $F=1 / 2$, the energies, $E$, are determined by the relations (see the supplementary material)

$$
\begin{aligned}
& \frac{j_{l}\left(k a_{e f f}\right)}{j_{|1-l|}\left(k a_{e f f}\right)}=(-1)^{l+1} \sqrt{\frac{\hbar^{2}}{2 m_{0}}} \sqrt{\frac{E_{p}}{3}} \frac{k}{\left(E_{v}-E\right)}, \text { (conduction band) } \\
& \frac{j_{l}\left(k a_{e f f}\right)}{j_{|1-l|}\left(k a_{e f f}\right)}=(-1)^{l+2} \sqrt{\frac{\hbar^{2}}{2 m_{0}}} \sqrt{\frac{E_{p}}{3}} \frac{k}{\left(E_{c}-E\right)} \quad \text { (valence band), }
\end{aligned}
$$

where for the $s$-like ground quantum size level $l=0$ and for the first excited $p$-like states $l=1$. Note that these expressions represent the fact that the $s$-like state in the conduction band is coupled to a $p$-like state in the valence band and vice versa. The quasimomenta $k$ in Eq. (5) are determined by the bulk band dispersion relation,

$$
\left(E_{c}-E\right)\left(E_{v}-E\right)=\frac{1}{3} \frac{\hbar^{2}}{m_{0}^{2}} P^{2} k^{2}=\frac{E_{p}}{3} \frac{\hbar^{2}}{2 m_{0}} k^{2} .
$$

In Eqs. (5) and (6), the Kane energy $E_{p}$ is given by

$$
E_{p}=2|P|^{2} / m_{0}
$$

where the Kane momentum matrix element $P=-i\langle s|\hat{P}| Z\rangle$ and $m_{0}$ is the free electron mass. To connect this picture to the simple effective mass theory, we note that in the 2-band model the band edge effective masses for the conduction and valence bands are given by ${ }^{19}$

$$
\frac{m_{0}}{m_{c}}=\frac{m_{0}}{m_{v}}=\frac{E_{p}}{3 E_{g}} .
$$

In Fig. 1, we show the confinement energy for the ground, $s$-like, and first excited, $p$-like, quantum size levels in the conduction band of $\mathrm{CsPbr}_{3}$ nanocrystals, using the experimentally derived effective mass parameters from Ref. 20 which are summarized in Table I. The comparison of the confinement energies calculated 
(a)

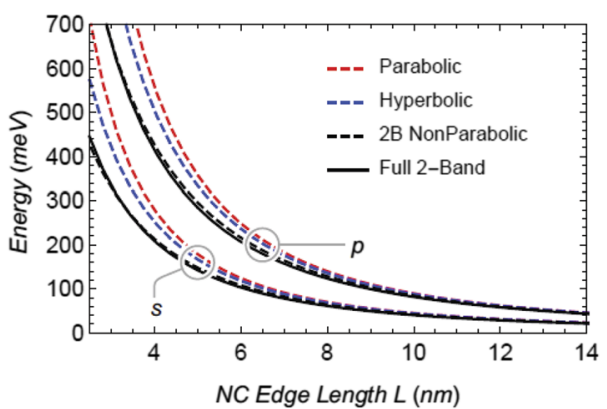

(b)

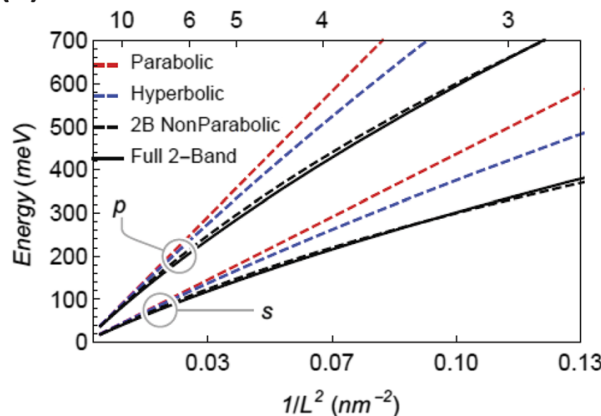

FIG. 1. Electron confinement energy $E-E_{c}$ for the ground $s$-like and first excited $p$-like quantum confined states in $\mathrm{CsPbBr} \mathrm{NCs}_{3} \mathrm{~N}$. In panel (a), the confinement energy relative to the conduction band edge is plotted vs edge length, $L$, of a cube-shaped NC, while in panel (b) the confinement energy is plotted vs the inverse square of the NC edge length. The solid black lines show the results of the full 2-band calculation, Eq. (5). Also shown for comparison are the calculated energies in the parabolic band approximation [Eq. (2) for the s-state, Eq. (4) for the p-state, red dashed lines], the hyperbolic band approximation of Ref. 27, [Eqs. (10) and (11), blue dashed lines], and finally, the "s/p" nonparabolic approximation derived here in Eqs. (12) and (13) (black dashed line). In the calculations, we used bandgap $E_{g}=2.342$ eV and Kane energy $E_{p}=27.88 \mathrm{eV}$ which correspond to the low temperature bandgap and effective mass measured experimentally for $\mathrm{CsPbBr}_{3}$ in the work of $\mathrm{Yang}_{\mathrm{P}}$ et al.; ${ }^{20}$ see Table I.

using the parabolic band model, Eq. (2), with the 2-band result, shown in Fig. 1, reveals that the two-band model has significantly lower confinement energy than that calculated in the parabolic model. In part, this is due to the nonparabolic dispersion of the bulk bands in the two-band model. This can be accounted for in principle by employing an energy-dependent effective mass. We illustrate this for the conduction band states. Starting with the 2-band dispersion relation, Eq. (6), we rearrange to obtain the expression

$$
E-E_{c}=\frac{\hbar^{2}}{2 m_{0}} \frac{E_{p}}{3\left(E-E_{v}\right)} k^{2}=\frac{\hbar^{2}}{2 m_{0}} \frac{m_{0}}{m_{c}(E)} k^{2},
$$

where the energy-dependent effective mass $m_{0} / m_{c}(E) \equiv E_{p} / 3\left(E-E_{v}\right)$ reflects the hyperbolic bulk band dispersion. The energy-dependent effective mass defined in the last equation reflects the hyperbolic bulk band dispersion. When implemented in single band effective mass theory, it leads to the following hyperbolic expression for the confinement energy, $\mathcal{E}_{s}=E-E_{c}$, of the ground state developed by Wang et al. in Ref. 27,

$$
\begin{gathered}
\mathcal{E}_{s}=\frac{\hbar^{2}}{2 m_{0}} \frac{E_{p}}{3\left(E_{g}+\mathcal{E}_{s}\right)}\left(\frac{\pi}{a_{e f f}}\right)^{2}=\frac{\hbar^{2}}{2 m_{0}} \frac{E_{p}}{3\left(E_{g}+\mathcal{E}_{s}\right)} 3\left(\frac{\pi}{L}\right)^{2}, \\
\therefore \mathcal{E}_{s}=\frac{-E_{g}+\sqrt{E_{g}^{2}+\frac{2 E_{p}}{m_{0}} \hbar^{2}\left(\frac{\pi}{L}\right)^{2}}}{2} .
\end{gathered}
$$

The corresponding expression for the excited $p$-state is, with $\mathcal{E}_{p}=E-E_{c}$,

$$
\begin{gathered}
\mathcal{E}_{p}=\frac{\hbar^{2}}{2 m_{0}} \frac{E_{p}}{3\left(E_{g}+\mathcal{E}_{p}\right)}\left(\frac{\alpha_{1,1}}{a_{e f f}}\right)^{2} \approx \frac{\hbar^{2}}{2 m_{0}} \frac{E_{p}}{3\left(E_{g}+\mathcal{E}_{p}\right)} 6\left(\frac{\pi}{L}\right)^{2}, \\
\therefore \mathcal{E}_{p}=\frac{-E_{g}+\sqrt{E_{g}^{2}+\frac{4 E_{p}}{m_{0}} \hbar^{2}\left(\frac{\pi}{L}\right)^{2}}}{2} .
\end{gathered}
$$

TABLE I. Summary of electronic structure parameters used in calculating the quantum size levels and exciton energy vs size of $\mathrm{CsPbBr}_{3} \mathrm{NCs}$ in Figs. 1 and 3. The first column shows the low temperature experimental values for bulk orthorhombic $\mathrm{CsPbBr}_{3}$ reported by Yang et al. in Ref. 20. The Kane energy is determined using Eq. (8) assuming that the effective masses of the conduction and valence bands are equal. For comparison to the room temperature $\mathrm{CsPbBr}_{3} \mathrm{NC}$ absorption data of Brennan et al. ${ }^{23}$ the Kane energy and dielectric are constrained to the reported values, while the bandgap (and with it the reduced effective mass) is determined by the best fit to the data, as shown in Fig. 3.

\begin{tabular}{lcc}
\hline \hline & $\begin{array}{c}\text { Experimental value }(2 \mathrm{~K}) \\
\text { from the work of } \\
\text { Yang et al., Ref. } 20\end{array}$ & $\begin{array}{c}\text { Best fit } \\
\text { model (300 K) }\end{array}$ \\
\hline Bandgap $E_{g}(\mathrm{eV})$ & 2.342 & 2.454 \\
Exciton reduced mass $\mu / m_{0}$ & 0.126 & 0.132 \\
Kane energy $E_{p}=\frac{3 E_{g} m_{0}}{2 \mu}(\mathrm{eV})$ & 27.88 & 27.88 (constrained) \\
Dielectric $\epsilon_{\text {eff }}$ & 7.3 & 7.3 (constrained) \\
\hline Exciton radius $a_{x}(\mathrm{~mm})$ & 3.07 & 2.93 \\
Exciton binding energy $B_{x}(\mathrm{meV})$ & 32.2 & 33.7 \\
\hline \hline
\end{tabular}


Using these hyperbolic expressions results in confinement energies which approach the full 2-band result more closely than the simple parabolic band approximation, as shown in Fig. 1, but still systematically overestimate the confinement energy calculated in the full two-band model.

The reason for the remaining discrepancy is that in the full two-band model, the ground conduction band state, which is predominantly $s$-like, is coupled to the $p$-like valence band state and vice versa for the ground valence band state [see Eq. (5)]. As a result, the dispersion relation which governs the quantum confined levels differs from the bulk dispersion relation, Eq. (9). In the case of the conduction band $s$-state, we approximate the difference by replacing the term $E_{v}$ in Eq. (9) by the energy of the lowest $p$-like valence band quantum size level, namely, $E_{v}-\mathcal{E}_{p}$, where $\mathcal{E}_{p}$ is taken from Eq. (11). For the excited $p$-like conduction band state, which couples to the $s$-like valence band state, we use the replacement $E_{v}-\mathcal{E}_{s}$, where $\mathcal{E}_{s}$ is taken from Eq. (10). This leads to the modified nonparabolic expressions for the confinement energies of the ground and first excited states, respectively, denoted as $\mathcal{E}_{s}^{\prime}$ and $\mathcal{E}_{p}^{\prime}$,

$$
\begin{aligned}
& \mathcal{E}_{s}^{\prime}=\frac{\hbar^{2}}{2 m_{0}} \frac{m_{0}}{m^{\prime}} 3\left(\frac{\pi}{L}\right)^{2}, \\
& \mathcal{E}_{p}^{\prime}=\frac{\hbar^{2}}{2 m_{0}} \frac{m_{0}}{m^{\prime}} 6\left(\frac{\pi}{L}\right)^{2},
\end{aligned}
$$

where the energy-dependent effective mass, $m^{\prime}$, is given in this " $s / p$ " nonparabolic approximation as

$$
\frac{m_{0}}{m^{\prime}} \equiv \frac{E_{p}}{3\left(E_{g}+\mathcal{E}_{s}+\mathcal{E}_{p}\right)} .
$$

In the last expression, we use Eqs. (10) and (11) for $\mathcal{E}_{s}$ and $\mathcal{E}_{p}$ to obtain

$$
\frac{m^{\prime}}{m_{0}} \equiv \frac{3\left(\sqrt{E_{g}^{2}+\frac{2 E_{p}}{m_{0}} \hbar^{2}\left(\frac{\pi}{L}\right)^{2}}+\sqrt{E_{g}^{2}+\frac{4 E_{p}}{m_{0}} \hbar^{2}\left(\frac{\pi}{L}\right)^{2}}\right)}{2 E_{p}} .
$$

Note that, unlike in the hyperbolic approximation, Eqs. (10) and (11), the expressions for the energies of the $s$ - and $p$-like states in Eqs. (12) and (13) now both involve the same energy-dependent effective mass, $m^{\prime}$, defined in Eq. (15). Remarkably, using this expression for the nonparabolic effective mass reproduces the full two-band result very closely in contrast to either the simple parabolic band approximation or the hyperbolic approximation, as shown in Fig. 1.

\section{EXCITONS IN MHP NANOCRYSTALS}

Having developed expressions for the quantum size levels of electrons and holes in MHP nanocrystals in Sec. II, we now consider the problem of excitons. We begin with a parabolic band approximation as before.

As discussed previously, cube-shaped MHP nanocrystals are now being fabricated with edge lengths $L$ ranging from a few nanometers to $\sim 15 \mathrm{~nm}$, that is, the grown sizes range from the exciton Bohr radius to several multiples of it. Such NCs are in an intermediate confinement regime, ${ }^{16,19}$ where confinement effects on the carrier energies are significant but the motion of the electron and hole are correlated. To capture the correlated motion of the electron and hole, we write the envelope function of the confined excitons using a one-parameter ansatz function, ${ }^{16,19}$

$$
f\left(\boldsymbol{r}_{e}, \boldsymbol{r}_{h}\right)=\frac{1}{\sqrt{N(\beta)}} e^{-\beta\left|\mathbf{r}_{e}-\mathbf{r}_{h}\right|} \psi_{\mathrm{gr}}\left(\boldsymbol{r}_{e}\right) \psi_{\mathrm{gr}}\left(\boldsymbol{r}_{h}\right) .
$$

Here, $\psi_{\mathrm{gr}}$ is the confined wavefunction found previously for carriers in the strong confinement limit, Eq. (1). This function satisfies the boundary condition that the envelope function vanishes at the NC surface, while the term involving $\beta$, the variational parameter, builds correlated electron-hole motion into the ansatz wavefunction. In the expression, $N(\beta)$ is a normalization factor. In the variational approach, we calculate the expectation value of the two-particle Hamiltonian,

$$
\hat{H}^{e f f}=-\frac{\hbar^{2}}{2 m_{e}} \nabla_{e}^{2}-\frac{\hbar^{2}}{2 m_{h}} \nabla_{h}^{2}-\frac{e^{2}}{\epsilon_{i n}\left|\boldsymbol{r}_{e}-\boldsymbol{r}_{h}\right|},
$$

where $\epsilon_{\text {in }}$ is the dielectric constant inside the NC. The procedure involves computing the kinetic energy [the expectation value of the first and second terms on the right-hand side of Eq. (17)] and the potential energy [the expectation value of the third term on the righthand side of Eq. (17)]. Expressing these as dimensionless integrals (given in Ref. 19), we can write the exciton energy $E_{x}$ relative to the bandgap, $E_{g}$, as

$$
E_{x}(L, b)=E_{g}-\frac{\hbar^{2}}{L^{2}}\left[\frac{1}{2}\left(\frac{1}{m_{e}}+\frac{1}{m_{h}}\right) \frac{I_{K}(b)}{N(b)}\right]-\frac{e^{2}}{\epsilon_{i n} L} \frac{I_{C}(b)}{N(b)} .
$$

For a given NC size $L$, we minimize the energy with respect to the dimensionless variational parameter $b=\beta$ L to obtain the exciton energy $E_{x}\left(L, b_{\text {opt }}\right)$, as described in Refs. 16 and 19 . We find that the ground state exciton energy smoothly varies between the strong confinement result when $L<a_{x}$,

$$
\begin{aligned}
E_{x}^{S t r}(L) & =E_{g}+\frac{\hbar^{2}}{2 \mu}\left(\frac{3 \pi^{2}}{L^{2}}\right)-3.05 \frac{e^{2}}{\epsilon_{i n} L}, \\
& =E_{g}+B_{x}\left(\frac{3 \pi^{2}}{\left(L / a_{x}\right)^{2}}-2 \times(3.05) \frac{a_{x}}{L}\right),
\end{aligned}
$$

and the weak confinement result when $L \gg a_{x}$,

$$
E_{x}^{\text {Weak }}(L)=E_{g}+B_{x}\left\{\frac{\mu}{M_{x}}\left(\frac{3 \pi^{2}}{\left(L / a_{x}\right)^{2}}\right)-1\right\} .
$$

In these expressions, the exciton binding energy, $B_{x}$, is given in terms of the hydrogen Rydberg, $R y$, as

$$
B_{x}=\frac{\mu}{m_{0} \epsilon_{i n}^{2}} R y=\frac{\hbar^{2}}{2 \mu a_{x}^{2}}=\frac{1}{2} \frac{e^{2}}{\epsilon_{i n} a_{x}} .
$$

$\mu=m_{e} m_{h} /\left(m_{e}+m_{h}\right)$ is the reduced effective mass given in terms of the electron and hole masses, $m_{e}$ and $m_{h}$, and $M_{x}=m_{e}+m_{h}$ is the total exciton effective mass.

The exciton energy calculated in these limits for parabolic band dispersion, with energy independent electron and hole masses $m_{e}$ and $m_{h}$, is shown in Fig. 2. In the figure, energy is given in units 


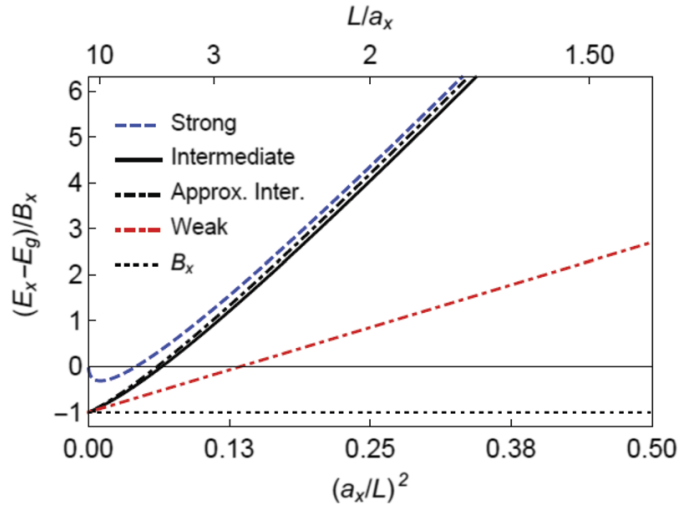

FIG. 2. Exciton energy $E_{x}-E_{g}$ plotted in units of the exciton binding energy, $B_{x}$, vs $\left(a_{x} / L\right)^{2}$, where $L$ is the edge length of a cube-shaped NC and $a_{x}$ is the exciton radius. The solid black line represents the result of a variational calculation valid for the intermediate confinement limit, $L \sim a_{x}$, Eq. (18). The black dashed line labeled "Approx. Inter." shows the intermediate confinement energy evaluated using the approximate expression given in Eq. (23). Also shown for reference are the exciton energies calculated in the strong confinement limit $\left[L \ll a_{x}\right.$, blue dashed line, Eq. (19)], the weak confinement limit [ $L \gg a_{x}$, red dashed line, Eq. (20)], and the exciton binding energy $B_{x}$ (black dashed line)

of the exciton binding energy $B_{x}$ relative to the bandgap $E_{g}$ and is plotted against the dimensionless quantity $\left(a_{x} / L\right)^{2}$. The energy in the intermediate confinement limit is shown in the figure as the solid black line. For small NC sizes, this converges as expected with the result calculated in the strong confinement limit, Eq. (19), plotted as the blue dashed line. For large sizes, it converges to the weak confinement limit, Eq. (20), shown as the red dashed line, approaching the exciton binding energy $B_{x}$ for large sizes.

From the plot, it is clear that for sizes as large as $L<\sim 3 a_{x}$, the intermediate confinement energy is very close to that calculated in the strong confinement limit. Indeed, a reasonable approximation to the energy in the intermediate confinement limit is to use the kinetic energy term associated with the strong confinement limit in Eq. (19) but to replace the Coulomb energy term [the third term on the right hand side in Eq. (19)] by the square root of the sum of the squares of the exciton binding energy $B_{x}$ and the Coulomb energy calculated in first order perturbation theory,

$$
E_{\text {Coul }}^{\text {Inter }}(L) \approx-B_{x} \sqrt{1+\left(2 \times(3.05) \frac{a_{x}}{L}\right)^{2}} .
$$

The resulting approximate expression for the exciton energy in intermediate confinement is given by

$$
E_{x}^{\text {Inter }} \approx E_{g}+B_{x}\left(\frac{3 \pi^{2}}{\left(L / a_{x}\right)^{2}}-\sqrt{1+\left(2 \times(3.05) \frac{a_{x}}{L}\right)^{2}}\right) .
$$

The exciton energy calculated in Eq. (23) is shown in Fig. 2 as the black dashed line and is seen to represent an excellent approximation to the full variational calculation shown in the figure.

The consideration of excitonic effects above was conducted in the parabolic band approximation. To account for band nonparabolicity, we can take the reduced effective mass as energy-dependent, or equivalently as dependent on the NC size, $L$, using $\mu(L)^{-1}=2 / m^{\prime}(L)$, where Eq. (15) defines the effective nonparabolic mass in the Kane model. This approach allows us to use Eq. (23) for nonparabolic bands with $\mu(L)^{-1}=m_{e}(L)^{-1}+m_{h}(L)^{-1}$ and introducing the size dependence of exciton binding energy $B_{x}(L)=\mu(L) /\left(m_{0} \epsilon_{i n}^{2}\right) \cdot R y$.

\section{A. Comparison of exciton quantum confinement models to experimental data}

We now compare the models developed above to experimental data. In Fig. 3(a), we show the room temperature absorption (a)

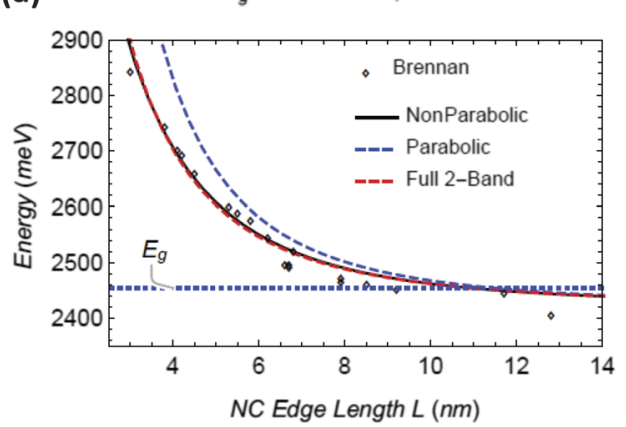

(b)

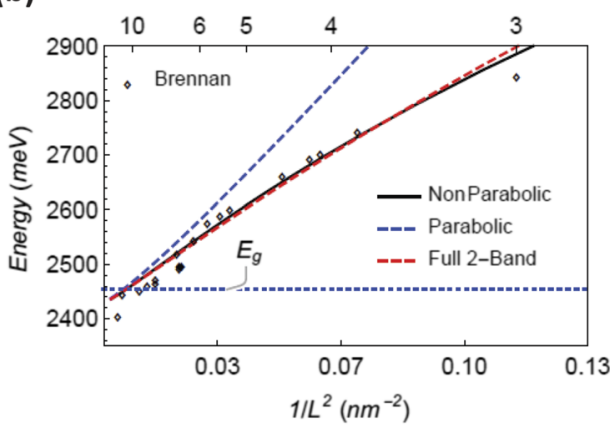

FIG. 3. $\mathrm{CsPbBr}_{3} \mathrm{NC}$ absorption peak energy vs $\mathrm{NC}$ size. Panel (a) shows room temperature absorption peak energy plotted vs edge length $\mathrm{L}$ for cube-shaped $\mathrm{CsPbBr}{ }_{3}$ NCs, while panel (b) shows the absorption peak energy plotted vs the inverse square of NC size L. The data points in the figure are from the work of Brennan et al. ${ }^{23}$ The theoretical curves shown are calculated using the $T=300 \mathrm{~K}$ parameters in Table I, which are taken from the $2 \mathrm{~K}$ magneto-transmission measurements on bulk $\mathrm{CsPbBr}_{3}$, Ref. 20, with the exception of the bandgap, $E_{g}$. $E_{g}$ was adjusted to achieve the best fit to the measured NC absorption data using the intermediate confinement model, Eq. (18), with the size-dependent nonparabolic effective mass given in Eq. (15); this calculation is plotted using solid black lines in the figure. For comparison, we also show by blue dashed lines the energy calculated within the parabolic band model described by Eq. (18) using the band edge effective mass and by red dashed lines the energy calculated within the full 2-band model using Eqs. (5) and (6) to determine the kinetic energy and the approximate expression [Eq. (22)] for the potential energy in intermediate confinement. 
peak energy of cube-shaped $\mathrm{CsPbBr}$. NCs measured as a function of nanocrystal edge length, $L$, reported by Brennan et al. in Ref. 23. Panel (b) of the figure shows the same data plotted vs $1 / L^{2}$ to emphasize the departure of the measured data from parabolic dispersion, which would show a linear dependence on $1 / L^{2}$. In the figure, we show the theoretical exciton energy calculated within the intermediate confinement approximation, corresponding to Eq. (18), for several approximations. First, the solid black line shows the intermediate confinement energy, Eq. (18), calculated within the nonparabolic model, using the size-dependent effective mass given in Eq. (15). The $T=300 \mathrm{~K}$ parameters used in this calculation are based on the measurements of Yang et al., ${ }^{20}$ summarized in Table I. The only parameter adjusted from their measured low temperature bulk values is the room temperature bandgap $E_{g}(300 \mathrm{~K})$ which was adjusted to achieve best fit to the measured room temperature $\mathrm{CsPbBr}_{3} \mathrm{NC}$ data of Ref. 23. To underscore the failure of the parabolic band approximation, we also show as a blue dashed line in the figure the exciton energy calculated for parabolic band dispersion, using Eq. (18) with the band-edge reduced exciton effective mass. This calculation only matches the data for the largest NC sizes; owing to significant band nonparabolicity, a parabolic dispersion model does not match the experimentally measured size dependence. Finally, for reference, we show the result of a full 2-band model, using Eqs. (5) and (6), to determine the kinetic energy and the approximate expression for the potential energy in intermediate confinement, given by Eq. (22). This calculation matches both the data of Brennan et al. and the nonparabolic effective mass model very well with only a slight departure in energy at the smallest NC sizes.

\section{EXCHANGE INTERACTION AND CRYSTAL FIELD SPLITTINC}

Having developed a model for the quantum confinement of excitons in MHP NCs in Sec. III, we now turn to the description of the exciton fine structure, that is, the millielectronvolt scale structure created by spin-dependent interactions between the electron and the hole. We start the discussion with the short-range (SR) exchange.

\section{A. Short-range exchange}

The SR exchange can be written in the form of a spindependent contact interaction, ${ }^{19,28}$

$$
\hat{H}_{\mathrm{exch}}^{S R}=\frac{1}{2} C \Omega\left[\mathbb{I}-\left(\boldsymbol{\sigma}_{e} \cdot \boldsymbol{\sigma}_{h}\right)\right] \delta\left(\boldsymbol{r}_{e}-\boldsymbol{r}_{h}\right) .
$$

In this expression, $\mathbb{I}$ is the $4 \times 4$ unit matrix, $\boldsymbol{\sigma}_{e}$ and $\boldsymbol{\sigma}_{h}$ are Pauli operators representing the electron and hole spin (true spin, not their total angular momenta), $\Omega$ is the volume of the crystal unit cell, while $C$ is the exchange constant. To obtain the size-dependent SR exchange energies, we simply average the exchange interaction over the total exciton wavefunction, including the underlying periodic part as well as the slowly varying envelope. We write the general form of the total exciton wavefunction, first, for a NC of cubic symmetry,

$$
\psi_{J_{e}, J_{h}}\left(\boldsymbol{r}_{e}, \boldsymbol{r}_{h}\right)=u_{J_{h}}^{v}\left(\boldsymbol{r}_{e}\right) u_{J_{e}}^{c}\left(\boldsymbol{r}_{h}\right) f\left(\boldsymbol{r}_{e}, \boldsymbol{r}_{h}\right)
$$

Here, $f\left(\boldsymbol{r}_{e}, \boldsymbol{r}_{h}\right)$ is the envelope function for the exciton which describes the electron and hole motion within the NC. Within the intermediate confinement regime, $f\left(\boldsymbol{r}_{e}, \boldsymbol{r}_{h}\right)$ is given by Eq. (16). The Bloch functions $u_{J_{e}}^{c}\left(\boldsymbol{r}_{e}\right)$ and $u_{J_{h}}^{v}\left(\boldsymbol{r}_{h}\right)$ represent the band-edge periodic basis functions for the exciton which contain the spin functions. For a cubic perovskite, as noted previously, the conduction and valence band states each have angular momentum $J=1 / 2$. For the valence band edge, which has $s$-symmetry, these are the even parity states of angular momentum $J=1 / 2$, which we write $u_{1 / 2, \pm 1 / 2}^{v}$, given by ${ }^{16}$

$$
u_{1 / 2,1 / 2}^{v}=S \uparrow, \quad u_{1 / 2,-1 / 2}^{v}=S \downarrow,
$$

where the spinor functions $\uparrow$ and $\downarrow$ are the eigenfunctions of the electron spin projection operator $s_{z}= \pm 1 / 2$. The spin-orbit interaction splits the conduction band, which has $p$-symmetry, into lower, band-edge states with angular momentum $J=1 / 2$ and upper states with $J=3 / 2$. The conduction band edge states $u_{1 / 2, \pm 1 / 2}^{c}$ are given by ${ }^{16}$

$$
\begin{gathered}
u_{1 / 2,1 / 2}^{c}=\frac{-1}{\sqrt{3}}[(X+i Y) \downarrow+Z \uparrow], \\
u_{1 / 2,-1 / 2}^{c}=\frac{1}{\sqrt{3}}[-(X-i Y) \uparrow+Z \downarrow],
\end{gathered}
$$

where $X, Y$, and $Z$ are the three orbital Bloch functions which transform as the functions having $p$ symmetry. Since there are two conduction band states and two valence band states, it is clear that the exchange interaction, described by Eq. (24), will take the form of a $4 \times 4$ matrix. Diagonalization of the resulting matrix shows that exchange splits the exciton into an upper, optically active triplet and a ground optically passive dark singlet. The size dependence of the exciton fine structure splitting can be obtained by averaging Eq. (24) over the wavefunction of the confined exciton. Since the envelope function in Eq. (25) is not itself spin-dependent, the result of this averaging takes the form

$$
\tilde{H}_{\text {exch }}^{S R}=C \tilde{M}^{S R} \Theta(L) .
$$

Here, $C$ is the exchange constant, $\tilde{M}^{S R}$ is a dimensionless matrix determined by the Bloch functions, Eqs. (26) and (27), while $\Theta(L)$ is the overlap factor representing the probability of the overlap of the electron and hole in the same unit cell,

$$
\begin{aligned}
\Theta(L) & =\Omega \iint_{V} d^{3} r_{e} d^{3} r_{h} f^{*}\left(\boldsymbol{r}_{e}, \boldsymbol{r}_{h}\right) \delta\left(\boldsymbol{r}_{e}-\boldsymbol{r}_{h}\right) f\left(\boldsymbol{r}_{e}, \boldsymbol{r}_{h}\right) \\
& =\Omega \int_{V} d^{3} r|f(\boldsymbol{r}, \boldsymbol{r})|^{2} .
\end{aligned}
$$

The integral above is taken over the volume $V=L^{3}$ of the cubeshaped NC with edge length $L$. Diagonalization of $\tilde{M}^{S R}$ for cubic perovskites yields a singlet level with eigenvalue 0 which corresponds to the dark exciton and 3 degenerate upper levels with eigenvalues equal to $2 / 3 C \Theta$ which are optically allowed. ${ }^{19}$ The size dependence of the splitting is given by the overlap factor $\Theta(L)$. Using the ansatz function, Eq. (16), in the intermediate confinement regime, we have

$$
\Theta_{\text {Inter }}=\frac{1}{N\left(b_{\text {opt }}\right)} \frac{27}{8} \frac{\Omega}{L^{3}},
$$


where $N\left(b_{o p t}\right)$ is the normalization calculated for each $L$ for the variational optimum parameter $b=b_{o p t}(L)$. We show this function in Fig. 4, normalized to the value of the overlap calculated in the bulk, $\Theta_{\text {bulk }}=\Omega / \pi a_{x}^{3}$, and plotted vs the ratio $L / a_{x}$ of the edge length, $L$, of a cube-shaped NC to the exciton radius $a_{x}$. The function $N\left(b_{\text {opt }}\right)$ $\rightarrow 1$ for $L \ll a_{x}$ recovering the well-known $1 / L^{3}$ dependence of the overlap $\Theta$ in the strong confinement limit. However, the exchange splitting calculated this way is seriously underestimated for $L>a_{x}$ because the correlated motion of the electron and hole is neglected in this limit since the overlap factor approaches the bulk value in the weak confinement limit $L \gg a_{x}: \Theta \rightarrow \Omega / \pi a_{x}^{3}$. Analytical expressions for the overlap in the strong, weak, and intermediate approximations are derived in Ref. 19.

For NCs with tetragonal and orthorhombic crystal structure, we expect that the 3 upper triplet levels will be split. The splitting is described by matrix $\tilde{M}^{S R}$, which will be different for tetragonal and orthorhombic lattice symmetry due to a modification of the Bloch functions of the conduction band edge, relative to those shown in Eq. (27). In Sec. IV A 1, we review the quasicubic model, which shows how the modified Bloch functions depend on the perovskite crystal symmetry for the tetragonal and orthorhombic phases, which serves as a starting point for our understanding of the fine structure.

\section{Quasicubic model for the crystal field}

In the quasicubic model, we consider the effect of the noncubic lattice structure as a simple distortion of the unit cell from the cubic phase. ${ }^{29}$ The approach is motivated by the observation that the lattice constants of the tetragonal and orthorhombic phases are typically within $\approx 1-2 \%$ of those of the cubic $\alpha$ phase, viewed in a nonprimitive supercell aligned to the tetragonal or orthorhombic unit cell, as appropriate. Alternately, the unit cell parameters of the noncubic phases can be expressed in "normalized" form in terms of an equivalent distortion of the lattice parameters of the cubic lattice. ${ }^{33}$ Either way, the differences can be quantified by defining strains along the principle axes relative to the cubic lattice. Having defined the strains, we then parameterize the effect on the conduction band edge states using a deformation potential model. ${ }^{30}$ The strain deformation Hamiltonian is constructed using the theory of invariants as

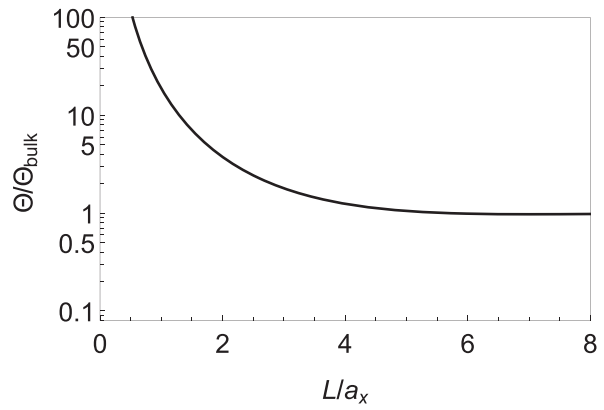

FIG. 4. Size dependence of the exchange splitting. The exchange overlap factor, $\Theta$, Eq. (29), is calculated vs NC size, $L$. In the plot, $\Theta$ is given in terms of the bulk overlap factor, $\Theta_{\text {bulk }}=\Omega /\left(\pi a_{x}^{3}\right)$, and is plotted vs the ratio $L / a_{x}$ of the edge length, $L$, of a cube-shaped $N C$ to the exciton radius $a_{x}$.

$$
\tilde{H}_{d}=U_{d}\left(e_{x x} L_{x}^{2}+e_{y y} L_{y}^{2}+e_{z z} L_{z}^{2}-2 / 3\left(e_{x x}+e_{y y}+e_{z z}\right) \mathbb{I}\right),
$$

where $U_{d}$ is a deformation potential; $e_{i i}$ are the components of the strain tensor with $i$ running over $x, y, z$, taken parallel to the crystal principal axes; and $L_{x, y, z}$ are the matrices representing the $x, y$, and $z$ projections of angular momentum $l=1$. Together with the $3 \times 3$ unit matrix, $\mathbb{I}$, the matrices $L_{x, y, z}$ serve as base matrices for the deformation potential Hamiltonian for the $p$-like conduction band with no spin-orbit coupling. Since we are only interested in the splitting caused within the conduction band manifold, we have subtracted off the volume dilatation in Eq. (31). In the basis $|1, \mathrm{~m}\rangle$ with $l=1$, taken in the order $|1,1\rangle,|1,0\rangle,|1,-1\rangle$, the deformation potential Hamiltonian then has the form

$$
\tilde{H}_{d}=U_{d}\left(\begin{array}{ccc}
\frac{1}{3}\left(\epsilon_{z z}-\frac{\epsilon_{x x}+\epsilon_{y y}}{2}\right) & 0 & \left.\frac{1}{2} \epsilon_{x x}-\epsilon_{y y}\right) \\
0 & -\frac{2}{3}\left(\epsilon_{z z}-\frac{\epsilon_{x x}+\epsilon_{y y}}{2}\right) & 0 \\
\frac{1}{2}\left(\epsilon_{x x}-\epsilon_{y y}\right) & 0 & \frac{1}{3}\left(\epsilon_{z z}-\frac{\epsilon_{x x}+\epsilon_{y y}}{2}\right)
\end{array}\right) .
$$

Equation (32) can be rewritten via symmetry-adapted strain combinations and we define the tetragonal, $\delta$, and orthorhombic, $\zeta$, crystal field parameters accordingly,

$$
\begin{aligned}
\delta & =U_{d} \epsilon_{\text {tet }}, & \zeta & =U_{d} \epsilon_{\text {ortho }}, \\
\epsilon_{\text {tet }} & =\epsilon_{z z}-\frac{\epsilon_{x x}+\epsilon_{y y}}{2}, & \epsilon_{\text {ortho }} & =\frac{\epsilon_{x x}-\epsilon_{y y}}{2} .
\end{aligned}
$$

For the valence bands, which are known to have $s$ symmetry, the only effect of the strain is a volume dilatation term-since the valence bands are orbitally quenched, there is no splitting as in the case of the conduction band; as a result, the valence band Bloch functions are unmodified in the quasicubic model.

To find the modified Bloch functions, we need to add spinorbit coupling, $\hat{H}_{S O}=\frac{2}{3} \Delta_{S O} \boldsymbol{L} \cdot \boldsymbol{S}$, to the quasicubic strain deformation model, where $L$ is the orbital angular momentum and $S$ is the spin. Then, it is straightforward to find the modified Bloch functions for the conduction and valence bands within a six-band model for the conduction electrons. For perovskites of tetragonal symmetry, these functions were found in Refs. 31 and 32 . This theory was extended in first order perturbation theory to the case of NCs with orthorhombic symmetry by Fu et al. ${ }^{12}$ In the supplementary material, we follow Fu et al. and show that the Bloch functions for the conduction band edge can be written approximately for all three perovskite crystal symmetries (cubic, tetragonal, and orthorhombic) as

$$
\begin{aligned}
u_{c 1}= & -\cos \phi \sin \theta Z \uparrow \\
& +\frac{(\sin \phi-\cos \phi \cos \theta) X-i(\sin \phi+\cos \phi \cos \theta) Y}{\sqrt{2}} \downarrow, \\
u_{c 2}= & \frac{(\sin \phi-\cos \phi \cos \theta) X+i(\sin \phi+\cos \phi \cos \theta) Y}{\sqrt{2}} \uparrow \\
& +\cos \phi \sin \theta Z \downarrow .
\end{aligned}
$$

Here, the terms $X, Y$, and $Z$ denote the orbital Bloch functions which transform as the functions having $p$ symmetry. The angles $\theta$ and $\phi$ are defined via the spin-orbit splitting, $\Delta_{\mathrm{SO}}$, of the conduction band 
and the tetragonal ${ }^{31,32}$ and orthorhombic ${ }^{12,14}$ crystal fields, $\delta$ and $\zeta$, respectively, as follows:

$$
\begin{gathered}
\tan 2 \theta=\frac{2 \sqrt{2} \Delta_{\mathrm{SO}}}{\Delta_{\mathrm{SO}}-3 \delta}, \quad \theta \leq \frac{\pi}{2}, \\
\tan 2 \phi=\frac{-4 \zeta \cos \theta}{\Delta_{\mathrm{SO}}+\delta+\sqrt{\Delta_{\mathrm{SO}}^{2}-(2 / 3) \Delta_{\mathrm{SO}} \delta+\delta^{2}}} .
\end{gathered}
$$

It is worth noting that in the case of cubic symmetry, $\delta=0, \zeta=0$ so that $\tan 2 \theta=2 \sqrt{2}$ or $\theta \approx 35.26^{\circ}$ and $\phi=0$. In this case, the conduction band Bloch functions reduce to those in Eq. (27). In the tetragonal case, $\zeta=0$ so that $\phi=0$, and therefore, the conduction band Bloch functions can be represented as eigenstates of the projection of total angular momentum along the c-axis, taken to be along $z^{31,32}$

The spin-orbit coupling parameter $\Delta_{\mathrm{SO}}$ can be obtained from experiments (by measuring the spacing between the conduction band edge states and the higher lying states of angular momentum $J=3 / 2$ ) or can be calculated using DFT (which is the approach taken later on in this paper). The crystal field parameters $\delta$ and $\zeta$ can also be determined by calculation using DFT. Absorption measurements can be used to determine the splitting as suggested in Ref. 14; however, if this approach is taken, polarization resolved measurements are required in order to distinguish which of the $J=3 / 2$ upper conduction band states moves upwards and which moves downwards under the action of the crystal field, which is required to determine the sign of the crystal field splitting. For example, in the tetragonal case, a positive crystal field causes the $J_{z}= \pm 3 / 2$ states, which are inactive for light polarized along the $\hat{z}$ axis, to move upwards in energy while the $J=3 / 2, J_{z}= \pm 1 / 2$ states, which are active for $\hat{z}$ polarized light, to move downwards. ${ }^{19}$ This trends become transparent in the no-spin-orbit coupling case, where the $Z$ state is shifted downwards relative to the $X$ and $Y$ orbital states for a positive crystal field; see Eqs. (32) and (33).

Averaging Eq. (24) over the four conduction and valence band Bloch function products, $\left|c_{1}\right\rangle\left|v_{\uparrow}\right\rangle,\left|c_{1}\right\rangle\left|v_{\downarrow}\right\rangle,\left|c_{2}\right\rangle\left|v_{\uparrow}\right\rangle$, and $\left|c_{2}\right\rangle\left|v_{\downarrow}\right\rangle$, we obtain the short-range exchange operator in the same from as Eq. (28), but now the matrix $\tilde{M}^{S R}=\tilde{M}^{S R}(\theta, \phi)$ is a function of the Bloch function phase angles which encode the symmetry. This matrix is given by

$$
\tilde{M}^{S R}(\theta, \phi)=\left(\begin{array}{cccc}
\cos ^{2} \theta \cos ^{2} \phi+\sin ^{2} \phi & 0 & 0 & \cos \theta \sin 2 \phi \\
0 & \cos ^{2} \phi \sin ^{2} \theta & \cos ^{2} \phi \sin ^{2} \theta & 0 \\
0 & \cos ^{2} \phi \sin ^{2} \theta & \cos ^{2} \phi \sin ^{2} \theta & 0 \\
\cos \theta \sin 2 \phi & 0 & 0 & \cos ^{2} \theta \cos ^{2} \phi+\sin ^{2} \phi
\end{array}\right) .
$$

Within the exchange model, the matrix $\tilde{M}^{S R}(\theta, \phi)$ again determines the level order and the oscillator transition strengths of each level, independently of the envelope wavefunction of the confined exciton. Diagonalizing the matrix $\tilde{M}^{S R}$ in Eq. (37) for angles $\theta$ and $\phi$ defined in Eqs. (35) and (36) results in energies for each exciton eigenstate $X_{i}$, where $X_{i}=\{X, Y, Z, D\}$, expressed in the general form

$$
E_{X_{i}}(\theta, \phi)=C \Theta \tilde{E}_{X_{i}}(\theta, \phi)=\frac{3}{2} \hbar \omega_{S T} \tilde{E}_{X_{i}}(\theta, \phi)\left(\frac{\Theta}{\Theta_{\text {bulk }}}\right) .
$$

Here, the energy scale is set by $C \Theta$, while $\tilde{E}_{X_{i}}(\theta, \phi)$ is a dimensionless eigenvalue of the matrix $\tilde{M}^{S R}$. Note that in the equation above, we have made the replacement, $\hbar \omega_{s t}=2 / 3 C \Theta_{b u l k}$, where $\hbar \omega_{s t}$ is the bulk exciton singlet-triplet splitting and $\Theta_{b u l k}=\Omega /\left(\pi a_{x}^{3}\right)$ is the overlap factor for the bulk. The functions $\tilde{E}_{X_{i}}$ are easy to evaluate and are given within the $\boldsymbol{k} \cdot \boldsymbol{P}$ model in Table II for the cubic, tetragonal, and orthorhombic phases. The average of $\tilde{E}_{X_{i}}(\theta, \phi)$ taken over the 3 triplet states $X, Y, Z$ is $2 / 3$ in all cases, as shown in the table. As a result, the average singlet-triplet splitting always equals $2 / 3 C \Theta$. In the cubic and tetragonal phases, $\phi=0$, while $\phi \neq 0$ in the orthorhombic phase.

The transition dipole matrix elements for each exciton sublevel are found as the matrix element of the momentum operator, $\hat{\boldsymbol{p}}$, between the exciton eigenfunction, $\psi_{X_{i}}$, and the crystal ground state,
$G$. The result can be expressed for each exciton eigenstate, $X_{i}$, in the general form ${ }^{19}$

$$
\boldsymbol{P}_{X_{i}}(\theta, \phi)=\mathcal{O} \boldsymbol{p}_{X_{i}}(\theta, \phi)=\mathcal{O} P \tilde{\boldsymbol{p}}_{X_{i}}(\theta, \phi),
$$

where $P$ is the Kane momentum matrix element defined above, $\tilde{\boldsymbol{p}}_{X_{i}}(\theta, \phi)$ is a dimensionless function describing the dependence of the matrix element on the crystal structure, and $\mathcal{O}$ is an overlap integral between the electron and hole wavefunctions, given by

$$
\mathcal{O} \equiv \iint_{V} f\left(\boldsymbol{r}_{e}, \boldsymbol{r}_{h}\right) \delta\left(\boldsymbol{r}_{e}-\boldsymbol{r}_{h}\right) d^{3} r_{e} d^{3} r_{h}=\int_{V} f(\boldsymbol{r}, \boldsymbol{r}) d^{3} r,
$$

where the integral is taken over the entire NC volume $V$. Analytical expressions for $\tilde{\boldsymbol{p}}_{X_{i}}(\theta, \phi)$ for NCs with different crystal symmetriescubic, tetragonal, and orthorhombic-are shown in Table II. Using the dipole matrix elements defined in Eq. (39), we can calculate the oscillator strength for each exciton state $X_{i}$,

$$
f_{X_{i}}=\mathcal{O}^{2} f_{0} \tilde{f}_{X_{i}}(\theta, \phi),
$$

where $f_{0}=E_{p} / \hbar \omega$ is the oscillator transition strength of a free carrier transition at the band edge, approximately equal to $f_{0} \approx E_{p} / E g$, $\mathcal{O}$ is the overlap integral [see Eq. (40)], and finally, $\tilde{f}_{X_{i}}=\left|\tilde{\boldsymbol{p}}_{X_{i}}(\theta, \phi)\right|^{2}$ is a reduced oscillator strength function that determines the relative 
TABLE II. Dimensionless energy, $\tilde{E}_{X_{i}}(\theta, \phi)$, and the dimensionless transition dipole matrix elements (DMEs) $\tilde{\boldsymbol{p}}_{X_{i}}$ for each exciton eigenstate $X_{i}$ in perovskite NCs with cubic, tetragonal, and orthorhombic crystal structure. $\tilde{\boldsymbol{p}}_{X_{i}}$ are expressed in the vector format $\left\{\tilde{p}_{x}, \tilde{p}_{y}, \tilde{p}_{z}\right\}$, where the $x, y, z$ axes are aligned to the $\mathbf{a}, \mathbf{b}, \mathbf{c}$ axes of the respective unit cell. The order of levels in the table is shown for a positive crystal fields $\delta$ and $\zeta$ with $\zeta<\delta$.

\begin{tabular}{|c|c|c|c|c|c|c|}
\hline \multirow[b]{2}{*}{ State $\left(X_{i}\right)$} & \multicolumn{2}{|c|}{ Cubic } & \multicolumn{2}{|c|}{ Tetragonal } & \multicolumn{2}{|c|}{ Orthorhombic } \\
\hline & Energy $\left(\tilde{E}_{X_{i}}\right)$ & $\operatorname{DME}\left(\tilde{\boldsymbol{p}}_{X_{i}}\right)$ & Energy $\left(\tilde{E}_{X_{i}}\right)$ & $\operatorname{DME}\left(\tilde{\boldsymbol{p}}_{X_{i}}\right)$ & Energy $\left(\tilde{E}_{X_{i}}\right)$ & $\operatorname{DME}\left(\tilde{\boldsymbol{p}}_{X_{i}}\right)$ \\
\hline $\mathrm{Z}$ & $\frac{2}{3}$ & $\sqrt{\frac{2}{3}}\{0,0,1\}$ & $2 \sin ^{2} \theta$ & $\{0,0, \sqrt{2} \sin \theta\}$ & $2 \cos ^{2} \phi \sin ^{2} \theta$ & $\{0,0, \sqrt{2} \cos \phi \sin \theta\}$ \\
\hline $\mathrm{Y}$ & $\frac{2}{3}$ & $\sqrt{\frac{2}{3}}\{0, i, 0\}$ & $\cos ^{2} \theta$ & $i\{0, \cos \theta, 0\}$ & $(\sin \phi+\cos \theta \cos \phi)^{2}$ & $i\{0, \cos \theta \cos \phi+\sin \phi, 0\}$ \\
\hline $\mathrm{x}$ & $\frac{2}{3}$ & $\sqrt{\frac{2}{3}}\{1,0,0\}$ & $\cos ^{2} \theta$ & $\{\cos \theta, 0,0\}$ & $(\sin \phi-\cos \theta \cos \phi)^{2}$ & $\{\cos \theta \cos \phi-\sin \phi, 0,0\}$ \\
\hline Triplet ave $\tilde{f}$ & $\frac{2}{3}$ & $\mathrm{NA}$ & $\frac{2}{3}$ & NA & $\frac{2}{3}$ & NA \\
\hline Dark & 0 & $\{0,0,0\}$ & 0 & $\{0,0,0\}$ & 0 & $\{0,0,0\}$ \\
\hline
\end{tabular}

oscillator strength of each exciton sublevel. Notably, within the sixband $\boldsymbol{k} \cdot \boldsymbol{P}$ /quasicubic model for the conduction bands, $\tilde{f}_{X_{i}}$ is the same function of $\theta$ and $\phi$ as the function $\tilde{E}_{X_{i}}$ that appears in the expressions for the energy, Eq. (38). ${ }^{19}$ Later, in this paper, we will check the validity of this result for several materials $\left(\mathrm{CsPBr}_{3}, \mathrm{FAPbBr}_{3}\right.$, and methylammonium lead iodide, $\mathrm{MAPbI}_{3}$ ) using DFT. We find that while the relationship is approximately true for the tetragonal phases of these materials, the relationship typically fails for the more complicated orthorhombic phases.

\section{Estimation of fine structure level order}

Having developed the quasicubic model to describe the exciton fine structure created by lattice distortion, we will estimate the SR exchange constant and the crystal field parameters for several materials of interest. DFT will be used for these calculations as well as for the testing of the quasicubic model. We start however with a qualitative estimation of the effect of the lattice distortion within the quasicubic model.

Using the expressions in Table II for the energy of each exciton state $\tilde{E}_{X_{i}}$, and the definitions of the phase angles $\theta, \phi$ in Eqs. (35) and (36) in terms of the crystal field parameters $\delta, \zeta$ in Eq. (33), we can write expressions for the fine structure energies simply in terms of the lattice constants of each material. To do this, we note that the expressions for the exchange energies in Table II can be linearized for small crystal field parameters $\delta \ll \Delta_{S O}, \zeta \ll \Delta_{S O}$. The lattice distortion causes the dimensionless exciton energies to deviate from the average value $2 / 3$ by an amount $\Delta \tilde{E}_{Z, X, Y}=\tilde{E}_{Z, X, Y}-2 / 3$ which can be written as

$$
\begin{aligned}
& \Delta \tilde{E}_{Z} \approx \frac{8}{9} \frac{\delta}{\Delta_{S O}} \approx \frac{4}{9} \frac{U_{d}}{\Delta_{S O}}\left(2 \epsilon_{z z}-\epsilon_{x x}-\epsilon_{y y}\right), \\
& \Delta \tilde{E}_{Y} \approx-\frac{4}{9} \frac{\delta}{\Delta_{S O}}-\frac{4}{3} \frac{\zeta}{\Delta_{S O}} \approx \frac{4}{9} \frac{U_{d}}{\Delta_{S O}}\left(2 \epsilon_{y y}-\epsilon_{z z}-\epsilon_{x x}\right), \\
& \Delta \tilde{E}_{X} \approx-\frac{4}{9} \frac{\delta}{\Delta_{S O}}+\frac{4}{3} \frac{\zeta}{\Delta_{S O}} \approx \frac{4}{9} \frac{U_{d}}{\Delta_{S O}}\left(2 \epsilon_{x x}-\epsilon_{y y}-\epsilon_{z z}\right) .
\end{aligned}
$$

In Eq. (42), we have expressed the crystal field parameters $\delta$, $\zeta$ in terms of the deformation potential and the lattice strains relative to the cubic phase. It bears emphasis that the assumption underlying the model is that the crystal field can be described in terms of a single deformation potential. To the extent that this assumption is valid, the result above shows that we can predict the order of levels solely on the basis on the quasicubic strain, which is determined by the lattice constants of a given material.

Given a material with tetragonal or orthorhombic lattice structure, we must first determine the strain associated with each principle axis. To consider the electronic structure of the tetragonal or orthorhombic phases in the quasicubic approximation, we need to transfer from the primitive unit cell of the cubic phase to a nonprimitive cell aligned to the tetragonal or orthorhombic primitive cell. For example, for the orthorhombic lattice structure, it is convenient to transfer to a nonprimitive cell of the cubic lattice spanned by vectors $\boldsymbol{a}, \boldsymbol{b}, \boldsymbol{c}$ with dimensions $\sqrt{2} a_{0}: \sqrt{2} a_{0}: 2 a_{0}$, where $a_{o}$ is the cubic phase lattice constant, and the vectors $\boldsymbol{a}$ and $\boldsymbol{b}$ are rotated by $45^{\circ}$ to the cubic phase $\boldsymbol{x}, \boldsymbol{y}$ axes, while the vector $\boldsymbol{c}$ is aligned to the cubic phase $z$ axis and twice the length of the primitive vector. Equivalently, we can define scaled quasicubic lattice parameters for the tetragonal and orthorhombic phases which represent a distorted primitive cubic cell. ${ }^{33}$ These scaled cell parameters, $a_{i}$, are equal to $b_{i} / \gamma_{i}$, where $b_{i}$ are the lattice parameters of the given noncubic phase (tetragonal or orthorhombic) and the factor $\gamma_{i}$ is the dimension of the corresponding nonprimitive cubic cell in the absence of any lattice distortion from cubic symmetry. In the orthorhombic case, for example, $\gamma_{x}=\gamma_{y}=\sqrt{2}$, while $\gamma_{z}=2$. Finally, to eliminate volume dilatation, rather than computing the strain relative to the cubic phase, we can simply compute the quasicubic strain relative to the parameter $\bar{a}_{N}=\left(a_{x} a_{y} a_{z}\right)^{1 / 3}$, which is the lattice parameter of a primitive cubic cell with the same volume as the scaled noncubic cell volume,

$$
\epsilon_{i i}=\frac{a_{i}-\bar{a}_{N}}{\bar{a}_{N}} .
$$

In Table III, we show this analysis for tetragonal $\mathrm{CsPbBr}_{3}$ and $\mathrm{FAPbBr}_{3}$ both corresponding to space group $\mathrm{P} 4 / \mathrm{mbm}$, No. 127. Also for comparison, we analyze tetragonal $\mathrm{MAPbI}_{3}$ (space group $I 4 / \mathrm{mcm}$, No. 140). Assuming a positive deformation potential in Eq. (42) for all three materials, we expect that $\mathrm{CsPbBr}_{3}$ and $\mathrm{MAPbI}_{3}$ will have a fine structure with a singlet $Z$-polarized exciton higher in energy than the degenerate $X / Y$ polarized doublet, while $\mathrm{FAPbBr}_{3}$ 
TABLE III. Quasicubic (QC) analysis of tetragonal $\mathrm{CsPbBr}_{3}(P 4 / \mathrm{mbm}), \mathrm{FAPbBr}_{3}(P 4 / \mathrm{mbm})$, and $\mathrm{MAPb}_{3}(14 / \mathrm{mcm})$. Lattice parameters are given for the primitive tetragonal unit cell, from Refs. 33-35. Scaled lattice parameters labeled $a_{i}$ refer to a distorted primitive cubic cell; these are equal to $b_{i} / \gamma_{i}$, where $\gamma_{i}$ gives the ideal ratio of the tetragonal supercell to the primitive cubic cell in the absence of any lattice distortion from cubic symmetry. For each axis, the strain is referenced to the parameter $\bar{a}_{N}$, which is the lattice parameter of a primitive cubic cell with the same volume as the normalized tetragonal cell volume. The tetragonal strain $\epsilon_{t e t}=\epsilon_{c c}-1 / 2\left(\epsilon_{a a}+\epsilon_{b b}\right)$, as described in the text. In the last two rows, we give the level order of the exciton states ordered from high energy to low expected based on Eq. (42) within the QC model and the level order calculated using DFT; see Table V. In the case of $\mathrm{FAPbBr}_{3}$ and $\mathrm{MAPbl}_{3}$, the DFT calculation is performed on a mimic structure with the experimental lattice structure but with the organic cation replaced by Cs; see text.

\begin{tabular}{|c|c|c|c|c|c|c|c|c|c|c|c|c|}
\hline & \multicolumn{4}{|c|}{$\mathrm{P} 4 / \mathrm{mbm}-\mathrm{Cs} \mathrm{PbBr}_{3}$} & \multicolumn{4}{|c|}{$P 4 / m b m-\mathrm{FAPbBr}_{3}$} & \multicolumn{4}{|c|}{$I 4 / m c m-\mathrm{MAPbI}_{3}$} \\
\hline & $b_{i}(\mathrm{~nm})$ & $\gamma_{i}$ & $a_{i}(\mathrm{~nm})$ & $\frac{a_{i}-a_{N}}{a_{N}}$ & $b_{i}(\mathrm{~nm})$ & $\gamma_{i}$ & $a_{i}(\mathrm{~nm})$ & $\frac{a_{i}-a_{N}}{a_{N}}$ & $b_{i}(\mathrm{~nm})$ & $\gamma_{i}$ & $a_{i}(\mathrm{~nm})$ & $\frac{a_{i}-a_{N}}{a_{N}}$ \\
\hline $\mathrm{a}$ & 0.826 & $\sqrt{2}$ & 0.584 & $-1.14 \%$ & 0.837 & $\sqrt{2}$ & 0.5921 & $+0.01 \%$ & 0.877 & $\sqrt{2}$ & 0.620 & $-0.71 \%$ \\
\hline$b$ & 0.826 & $\sqrt{2}$ & 0.584 & $-1.14 \%$ & 0.837 & $\sqrt{2}$ & 0.5921 & $+0.01 \%$ & 0.877 & $\sqrt{2}$ & 0.620 & $-0.71 \%$ \\
\hline c & 0.605 & 1 & 0.605 & $+2.32 \%$ & 0.592 & 1 & 0.5919 & $-0.03 \%$ & 1.267 & 2 & 0.633 & $+1.43 \%$ \\
\hline$a_{N}$ & & & 0.591 & & & & 0.5920 & & & & 0.624 & \\
\hline$\epsilon_{\text {tet }}$ & \multicolumn{4}{|c|}{$+3.45 \%$} & \multicolumn{4}{|c|}{$-0.04 \%$} & \multicolumn{4}{|c|}{$+2.14 \%$} \\
\hline QC & \multicolumn{4}{|c|}{ Z, X/Y, D } & \multicolumn{4}{|c|}{ X/Y, Z, D } & \multicolumn{4}{|c|}{ Z, X/Y, D } \\
\hline DFT & \multicolumn{4}{|c|}{ Z,X/Y, D } & \multicolumn{4}{|c|}{ X/Y, Z, D } & \multicolumn{4}{|c|}{ Z,X/Y, D } \\
\hline
\end{tabular}

is expected to have the opposite level order, namely, the $Z$-polarized exciton should lie below the $X / Y$ polarized doublet. Anticipating results to be developed later, we show in the table the level orders calculated using DFT for tetragonal $\mathrm{CsPbBr}_{3}$ as well as mimic structures corresponding to the experimental $\mathrm{FAPbBr}_{3}$ and $\mathrm{MAPbI}_{3}$ lattice structures in which the organic cations have been replaced by Cs. In each case, the level order agrees with expectations based on the quasicubic model, validating the qualitative applicability of the quasicubic model for the tetragonal structures as well as the assumption of a positive deformation potential constant.

The quasicubic analysis can be applied to the orthorhombic phases as well. In Table IV, we show the analysis for orthorhombic $\mathrm{CsPbBr} 3, \mathrm{FAPbBr}_{3}$, and $\mathrm{MAPbI}_{3}$, all characterized by space group
Pnma, No 62. Again assuming a positive deformation potential in Eq. (42) as was validated for the tetragonal phases for all three materials, the expected level order for orthorhombic $\mathrm{Cs} \mathrm{PbBr}_{3}$ and $\mathrm{FAPbBr}_{3}$ should be, from highest to lowest, $Z, Y, X$ above the ground dark state, while for $\mathrm{MAPbI}_{3}$ the order is expected within the quasicubic model to be $Y, Z, X, D$, as shown in the table. In each case however, the level order calculated using DFT for $\mathrm{CsPbBr}_{3}$ and the two mimic compounds disagrees with the order expected in the quasicubic model.

We turn in Sec. IV A 3 to the calculation of the exchange fine structure for tetragonal and orthorhombic $\mathrm{CsPbBr}_{3}, \mathrm{FAPbBr}_{3}$, and $\mathrm{MAPbI}_{3}$ using DFT, both to test the quasicubic model and to determine exchange constants and crystal field parameters that can be used for modeling the properties of NCs.

TABLE IV. QC analysis of orthorhombic ( $P n m a) C s \mathrm{PbBr}_{3}, \mathrm{FAPbBr}_{3}$, and $\mathrm{MAPbl}_{3}$. Lattice parameters $b_{i}$ are given for the primitive orthorhombic unit cell, from Refs. 33 , 36, and 37 , respectively. Scaled lattice parameters referred to a distorted primitive cubic cell labeled $a_{i}$; these are equal to $b_{i} / \gamma_{i}$, where $\gamma_{i}$ gives the ideal ratio of the orthorhombic cell to the primitive cubic cell in the absence of any lattice distortion from cubic symmetry. For each axis, the strain is referenced to the parameter $\bar{a}_{N}$, which is the lattice parameter of a primitive cubic cell with the same volume as the normalized tetragonal cell volume. The tetragonal and orthorhombic strains are calculated as described in the text. In the last two rows, we give the level order of the exciton states ordered from high energy to low expected based on Eq. (42) within the QC model and the level order calculated using DFT; see Table VI. In the case of $\mathrm{FAPbBr}_{3}$ and $\mathrm{MAPbl}_{3}$, the DFT calculation is performed on a mimic structure with the experimental lattice structure but with the organic cation replaced by Cs; see text. For all cases, the QC model fails to predict the level order correctly.

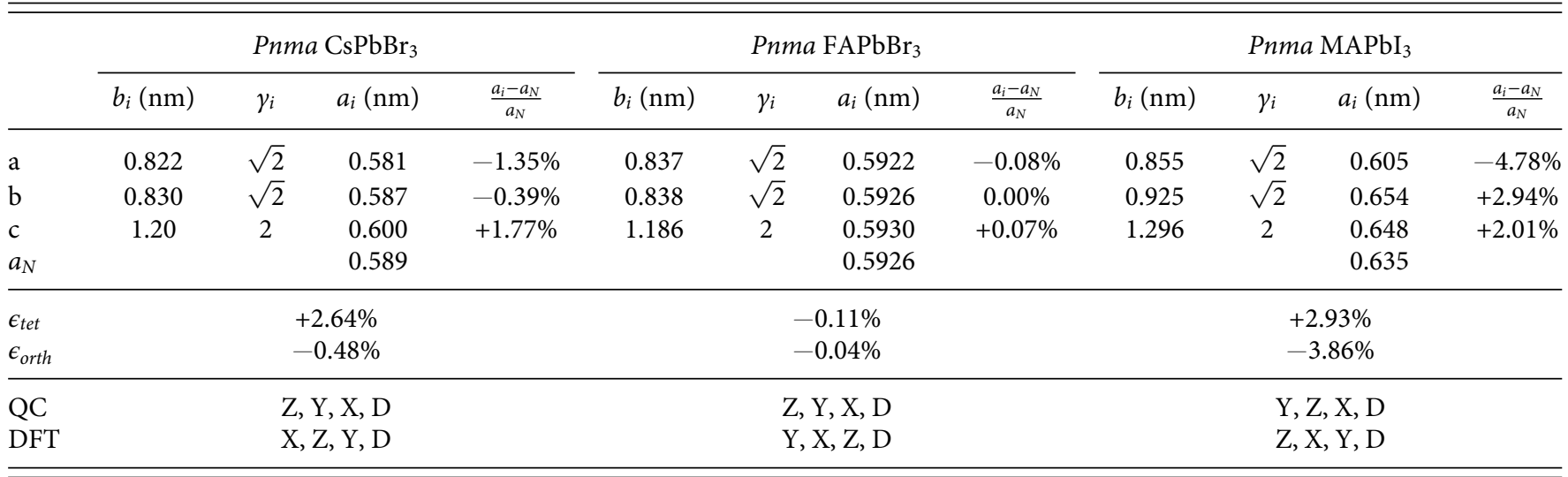




\section{Test of quasicubic model against DFT calculations}

In order to evaluate the validity of the quasicubic model as well as to determine exchange constants and crystal field parameters that can be used for modeling, we calculated the SR exchange Hamiltonian in a basis of the conduction- and valence-band-edge wavefunctions as determined in hybrid $\mathrm{DFT}^{38,39}$ as implemented in the VASP code ${ }^{40}$ for all the materials of interest. Diagonalization of the resulting exchange Hamiltonian enables determination of the bulk SR exchange constant $C^{S R}$ as well as the crystal field parameters. The details of this calculation are described in detail in the supplementary material and in Ref. 19.

We first calculated the SR exchange constants and crystal field parameters for tetragonal and orthorhombic $\mathrm{CsPbBr}_{3}$, using an experimental crystal structure reported in Ref. 33. In our calculations, all lattice parameters and atomic positions were subsequently relaxed to ensure self-consistency. These hybrid functional calculations yielded lattice parameters and energy gaps that are in good agreement with experiment $^{33,41}$ (see Table S1 of the supplementary material).

For a broader test of the quasicubic model encompassing different MHP lattice structures, we defined "mimic" FAPbBr 3 structures, comprising $\mathrm{CsPbBr}_{3}$ in tetragonal and orthorhombic structures with lattice constants and $\mathrm{Pb}$ and $\mathrm{Br}$ ion positions matching to those in the experimental $\mathrm{FAPbBr}_{3} \mathrm{P} 4 / \mathrm{mbm}$ structure reported in Ref. 34 and the Pnma structure reported in Ref. 36, respectively. The organic cations in these structures were replaced by Cs to preserve the inversion symmetry following the approach suggested in Ref. 24 for $\mathrm{MAPbI}_{3}$. Similarly, we defined mimic $\mathrm{MAPbI}_{3}$ structures, comprising $\mathrm{CsPbI}_{3}$ structures with lattice parameters taken from the experimental measurements of Refs. 35 and 37 for the tetragonal $(I 4 / \mathrm{mcm})$ and orthorhombic (Pnma) modifications, respectively, again with the replacement of the organic cation by Cs to preserve the inversion symmetry of the experimental structure. For these mimic structures, the Cs atoms were explicitly placed at the high-symmetry sites which were nearest to the center of the organic cations, and the atomic positions were not relaxed prior to calculation of the band structure and band edge wavefunctions.

Diagonalization of the exchange Hamiltonian enables determination of the bulk SR exchange constant $C^{S R}$ as well as the crystal field parameters. The results of these calculations are shown in Table $\mathrm{V}$ for the tetragonal phase of $\mathrm{CsPbBr}_{3}$ and the tetragonal $\mathrm{FAPbBr}_{3}$ and $\mathrm{MAPbI}_{3}$ mimic structures, and in Table VI for the corresponding orthorhombic phases. The SR exchange constant $C^{S R}$ is given for each phase along with the unit cell volume. Since the exchange constant scales as the inverse of the unit cell volume, it is useful to define a quasicubic exchange constant $C_{q c}^{S R}$ as the exchange constant multiplied by the ratio of the unit cell volume to the normalized cubic cell volume as defined in Table III. In each table, the reduced energy of each exciton state, $\tilde{E}_{X_{i}}$, is given, with the singlet state energy defined as the zero of energy.

In Sec. IV A 2 in discussing the quasicubic model, we anticipated the results for the reduced energies calculated in DFT shown in Tables $V$ and VI. To recap briefly, for all of the tetragonal

TABLE V. Short-range exchange constants, exciton energies, oscillator strengths, and crystal field parameters for tetragonal MHPs calculated using DFT with hybrid functionals The first column shows results for tetragonal $\mathrm{CsPbBr}_{3}$ calculated for the $\mathrm{P} 4 / \mathrm{mbm}$ structure given in Ref. 33 . The second column shows $\mathrm{CsPbBr}_{3}$ calculated for a mimic structure with lattice parameters matching that of $\mathrm{P} 4 / \mathrm{mbm} \mathrm{FAPbBr} 3$ from Ref. 34, while the third column shows $\mathrm{CsPb}_{3}$ in a mimic structure matching $\mathrm{MAPbl}_{3}$ from Ref. 35 , corresponding to space group $14 / \mathrm{mcm}$. The SR exchange constant $C^{S R}$ for each structure is determined by diagonalization of the SR exchange Hamiltonian using band-edge functions calculated in DFT. The quasicubic exchange constant $C_{q c}^{S R}$ is the exchange constant multiplied by the ratio of the unit cell volume to the normalized cubic cell volume, $\bar{a}_{N}^{3}$, where $\bar{a}_{N}$ is given for the corresponding structure in Table III. For each phase, the energy of each exciton state, $\tilde{E}_{X_{i}}$, calculated relative to the dark exciton energy, is given in units of $C^{S R} \Theta$, with the singlet state energy defined as the zero of energy $\left(E_{D}=0\right)$. Also given is the reduced oscillator strength, $\tilde{f}_{i}$; see Eq. (41). The $\boldsymbol{x}, \boldsymbol{y}, \boldsymbol{z}$ axes are defined as the a, b, $\mathbf{c}$ axes of the respective unit cell. The phase parameters $\theta$ and $\phi$ from Eq. (34), determined separately from the DFT exciton energies and oscillator strengths using the expressions in Table II, are shown in the table, as are the crystal fields determined using Eqs. (35) and (36) using spin-orbit coupling parameters $\Delta_{\text {so }}$ determined from DFT and given in the table.

\begin{tabular}{|c|c|c|c|c|c|c|}
\hline & \multicolumn{2}{|c|}{$P 4 / m b m \mathrm{CsPbBr}_{3}$} & \multicolumn{2}{|c|}{$P 4 / m b m \mathrm{CsPbBr}_{3}, \mathrm{FA}$ mimic } & \multicolumn{2}{|c|}{$I 4 / \mathrm{mcm} \mathrm{CsPbI}_{3}, \mathrm{MA}$ mimic } \\
\hline$C^{S R}$ & \multirow{3}{*}{\multicolumn{2}{|c|}{$\begin{aligned} C_{t e t}^{S R} & =223.4 \mathrm{meV} \\
\Omega_{t e t} & =0.4127 \mathrm{~nm}^{3} \\
C_{q c}^{S R} & =446.7 \mathrm{meV}\end{aligned}$}} & \multirow{3}{*}{\multicolumn{2}{|c|}{$\begin{aligned} C_{t e t}^{S R} & =254.8 \mathrm{meV} \\
\Omega_{t e t} & =0.4150 \mathrm{~nm}^{3} \\
C_{q c}^{S R} & =509.5 \mathrm{meV}\end{aligned}$}} & \multirow{3}{*}{\multicolumn{2}{|c|}{$\begin{aligned} C_{t e t}^{S R} & =101.5 \mathrm{meV} \\
\Omega_{\text {tet }} & =0.9740 \mathrm{~nm}^{3} \\
C_{q c}^{S R} & =406.1 \mathrm{meV}\end{aligned}$}} \\
\hline Cell vol. $\Omega$ & & & & & & \\
\hline & & & & & & \\
\hline State $\left(X_{i}\right)$ & $\tilde{E}_{X_{i}}$ & $\tilde{f}_{X_{i}}$ & $\tilde{E}_{X_{i}}$ & $\tilde{f}_{X_{i}}$ & $\tilde{E}_{X_{i}}$ & $\tilde{f}_{X_{i}}$ \\
\hline $\mathrm{Z}$ & 0.754 & 0.806 & 0.662 & 0.681 & 0.758 & 0.748 \\
\hline $\mathrm{Y}$ & 0.622 & 0.597 & 0.669 & 0.659 & 0.621 & 0.626 \\
\hline $\mathrm{X}$ & 0.622 & 0.597 & 0.669 & 0.659 & 0.621 & 0.626 \\
\hline Triplet ave & 0.667 & 0.667 & 0.667 & 0.667 & 0.667 & 0.667 \\
\hline $\operatorname{SOC} \Delta_{\mathrm{SO}}$ & \multicolumn{2}{|c|}{$1543 \mathrm{meV}$} & \multicolumn{2}{|c|}{$1460 \mathrm{meV}$} & \multicolumn{2}{|c|}{$1550 \mathrm{meV}$} \\
\hline Tetr. phase $\theta$ & $37.901^{\circ}$ & $39.408^{\circ}$ & $35.108^{\circ}$ & $35.702^{\circ}$ & $38.012^{\circ}$ & $37.707^{\circ}$ \\
\hline Tetr. CF $\delta$ & $+146.3 \mathrm{meV}$ & $+226.7 \mathrm{meV}$ & $-8.5 \mathrm{meV}$ & $+23.5 \mathrm{meV}$ & $+153.0 \mathrm{meV}$ & $+136.4 \mathrm{meV}$ \\
\hline
\end{tabular}


TABLE VI. Short-range exchange constants, exciton energy, oscillator strength, and crystal field parameters for orthorhombic MHPs calculated using DFT with hybrid functionals. The first column shows results for $\mathrm{CsPBBr}_{3}$ calculated in its Pnma structure given in Ref. 33. The second column shows $\mathrm{CsPbBr}_{3}$ calculated for a mimic structure with lattice parameters matching that of $\mathrm{Pnma} \mathrm{FAPbBr}_{3}$ from Ref. 36, while the third column shows $\mathrm{CsPbl}_{3}$ in a mimic structure matching $\mathrm{MAPb}_{3}$ from Ref. 37 . The SR exchange constant $C^{S R}$ for each structure is determined by diagonalization of the SR exchange Hamiltonian using band-edge functions calculated in DFT. The quasicubic exchange constant $C_{q c}^{S R}$ is the exchange constant multiplied by the ratio of the unit cell volume to the normalized cubic cell volume, $\bar{a}_{N}^{3}$, where $\bar{a}_{N}$ is given for the corresponding structure in Table IV. For each phase, the energy of each exciton state, $\tilde{E}_{X_{i}}$, calculated relative to the dark exciton energy, is given in units of $C^{S R} \Theta$, with the singlet state energy defined as the zero of energy $\left(E_{D}=0\right)$. Also given is the reduced oscillator strength, $\tilde{f}_{i}$; see (39). The $\boldsymbol{x}, \boldsymbol{y}, \boldsymbol{z}$ axes are defined as the $\mathbf{a}, \mathbf{b}, \mathbf{c}$ axes of the respective unit cell. The phase parameters $\theta$ and $\phi$ from Eq. (34), determined separately from the DFT exciton energies and oscillator strengths using the expressions in Table II, are shown in the table, as are the crystal fields determined using Eqs. (35) and (36) using spin-orbit coupling parameters $\Delta_{\mathrm{SO}}$ determined from DFT and given in the table.

\begin{tabular}{|c|c|c|c|c|c|c|}
\hline & \multicolumn{2}{|c|}{$P_{n m a} \mathrm{CsPbBr}_{3}$} & \multicolumn{2}{|c|}{$P_{n m a} \mathrm{CsPbBr}_{3}, \mathrm{FA}$ mimic } & \multicolumn{2}{|c|}{$P_{n m a} \mathrm{CsPbI}_{3}, \mathrm{MA} \mathrm{mimic}$} \\
\hline $\begin{array}{l}C^{S R} \\
\text { Cell vol. } \Omega \\
C_{q c}^{S R}\end{array}$ & \multicolumn{2}{|c|}{$\begin{aligned} C_{\text {orth }}^{S R} & =108.9 \mathrm{meV} \\
\Omega_{\text {orth }} & =0.8188 \mathrm{~nm}^{3} \\
C_{\text {cub }}^{S R} & =435.4 \mathrm{meV}\end{aligned}$} & \multicolumn{2}{|c|}{$\begin{aligned} C_{\text {orth }}^{S R} & =102.9 \mathrm{meV} \\
\Omega_{\text {orth }} & =0.8324 \mathrm{~nm}^{3} \\
C_{q c}^{S R} & =411.5 \mathrm{meV}\end{aligned}$} & \multicolumn{2}{|c|}{$\begin{array}{c}C_{\text {orth }}^{S R}=75.8 \mathrm{meV} \\
\Omega_{\text {orth }}=1.0261 \mathrm{~nm}^{3} \\
C_{q c}^{S R}=303.2 \mathrm{meV}\end{array}$} \\
\hline State $\left(X_{i}\right)$ & $\tilde{E}_{X_{i}}$ & $\tilde{f}_{X_{i}}$ & $\tilde{E}_{X_{i}}$ & $\tilde{f}_{X_{i}}$ & $\tilde{E}_{X_{i}}$ & $\tilde{f}_{X_{i}}$ \\
\hline $\mathrm{Z}$ & 0.663 & 0.699 & 0.597 & 0.809 & 0.726 & 0.795 \\
\hline $\mathrm{Y}$ & 0.628 & 0.620 & 0.744 & 0.645 & 0.571 & 0.570 \\
\hline $\mathrm{X}$ & 0.709 & 0.681 & 0.659 & 0.545 & 0.702 & 0.635 \\
\hline Triplet ave & 0.667 & 0.667 & 0.667 & 0.667 & 0.667 & 0.667 \\
\hline $\operatorname{SOC} \Delta_{\mathrm{SO}}$ & \multicolumn{2}{|c|}{$1543 \mathrm{meV}$} & \multicolumn{2}{|c|}{$1460 \mathrm{meV}$} & \multicolumn{2}{|c|}{$1550 \mathrm{meV}$} \\
\hline Tetr. phase $\theta$ & $35.162^{\circ}$ & $36.234^{\circ}$ & $33.139^{\circ}$ & $39.524^{\circ}$ & $37.099^{\circ}$ & $39.105^{\circ}$ \\
\hline Tetr. CF $\delta$ & $-5.5 \mathrm{meV}$ & $+54.7 \mathrm{meV}$ & $-118.2 \mathrm{meV}$ & $+220.3 \mathrm{meV}$ & $+103.1 \mathrm{meV}$ & $+211.6 \mathrm{meV}$ \\
\hline Orth. phase $\phi$ & $-1.387^{\circ}$ & $-1.089^{\circ}$ & $+1.463^{\circ}$ & $+1.864^{\circ}$ & $-2.361^{\circ}$ & $-1.206^{\circ}$ \\
\hline Orth. CF $\zeta$ & $+45.5 \mathrm{meV}$ & $+36.8 \mathrm{meV}$ & $-43.4 \mathrm{meV}$ & $-65.1 \mathrm{meV}$ & $+82.1 \mathrm{meV}$ & $+44.2 \mathrm{meV}$ \\
\hline
\end{tabular}

structures calculated, the level order calculated using DFT agrees with the expectations based on the quasicubic model. However, in all of the orthorhombic structures, the calculated level order disagrees with the analytical quasicubic model.

This discrepancy goes deeper than this however. Tables $V$ and VI also show the reduced oscillator strengths, $\tilde{f}_{X_{i}}$, calculated in DFT. This quantity is found from the band-edge transition oscillator strength $f_{X_{i}}$ using the relation $f_{X_{i}}=\left(E_{p} / \hbar \omega\right) \tilde{f}_{X_{i}}$, [see Eq. (41)]. In $\boldsymbol{k} \cdot \boldsymbol{P}$ theory, the reduced exciton energy $\tilde{E}_{X_{i}}$ of the $X_{i}$ exciton is equal to $\tilde{f}_{X_{i}}$. Inspection of Table $V$ shows that the relationship between $\tilde{f}_{X_{i}}$ and $E_{X_{i}}$ obtained by DFT qualitatively follows the $\boldsymbol{k} \cdot \boldsymbol{P}$ prediction for the tetragonal phases of $\mathrm{CsPbBr}_{3}$ and the mimic $\mathrm{MAPbI}_{3}$ structure; however, the relationship is not followed for the tetragonal mimic $\mathrm{FAPbBr}_{3}$ structure or for any of the orthorhombic structures. In these cases, the order of levels in energy differs from the order of levels ranked by oscillator strength. Nevertheless, in all cases, the averages of the quantities $\tilde{E}_{X_{i}}$ and $\tilde{f}_{X_{i}}$ over the triplet states $X, Y, Z$ are $2 / 3$ as expected within the quasicubic model.

To emphasize the departure of the DFT calculation from the quasicubic model, the crystal field parameters $\delta$ and $\zeta$ were calculated, first, using the reduced energies determined using DFT, and then second, for comparison, using the reduced oscillator strengths determined using DFT. This is a two step process. First, from the reduced energies, $\tilde{E}_{Z}$ and $\tilde{E}_{X}$, or the reduced oscillator strengths, $\tilde{f}_{Z}$ and $\tilde{f}_{X}$, calculated within DFT, we determine the phase angles $\theta$ and $\phi$ using the expressions in Table II. Then, using Eqs. (35) and (36), we determine $\delta$ and $\zeta$ in terms of the two phase angles $\theta$ and $\phi$ and the spin-orbit coupling $\Delta_{\text {SO }}$, also calculated by DFT. The values of the crystal field parameters calculated in this manner are shown for comparison in Tables V and VI.

For the tetragonal $\mathrm{CsPbBr}_{3}$ structure and the tetragonal $\mathrm{CsPbI}_{3}$ MA mimic, we find positive crystal fields when calculated either using the reduced energy or the reduced oscillator strength. However, the magnitudes of the crystal fields determined by the two methods are significantly different: In several cases, such as for the tetragonal $\mathrm{FAPbBr}_{3}$ mimic structure, and two of the three orthorhombic structures calculated, the crystal field has the opposite sign when calculated using the reduced energies vs the reduced oscillator strengths, which is a result that runs completely contrary to the analysis within the 6-band quasicubic model. 
As expected, this discrepancy between the DFT calculation and the quasicubic model is connected with the presence of higher conduction bands not included in the 6-band quasicubic conduction band model. The root of the failure has to do with the restricted basis within which the crystal field Hamiltonian, Eqs. (31)-(33), is written. Indeed, the quasicubic model completely fails to describe the relationship between the crystal field and the upper conduction band energies for tetragonal $\mathrm{CsPBr}_{3} .{ }^{19}$ In that case, in addition to the 6 conduction bands included in the quasicubic analysis, which are folded onto the Z-point of the tetragonal Brillouin zone (BZ) from the R-point of the cubic structure, there is an additional 2-fold degenerate band close in energy above the four $J=3 / 2$ states which originates from the $\mathrm{z}$-directed $\mathrm{X}$-point of the $\mathrm{BZ}$ of the cubic structure which couples with the R-point derived states. The resulting level repulsion increases the upper $J=3 / 2$ conduction band splitting by nearly a factor of $\sim 3$ above what is predicted in the quasicubic model. ${ }^{19}$ Moreover, preliminary results indicate that the increased oscillator strength ratio $\tilde{f}_{Z} / \tilde{f}_{X / Y}$ relative to the fine structure energy ratio $\tilde{E}_{Z} / \tilde{E}_{X / Y}$ in this structure is explained by coupling to the higher conduction band.

Despite the significant discrepancy between the DFT results and the quasicubic model, we can still employ the relations developed above within the effective mass theory for the SR exchange, such as Eq. (38). However, we must account for the fact that the reduced energy $\tilde{E}_{X_{i}}$ in Eq. (38) and the reduced oscillator strength $\tilde{f}_{X_{i}}$ in Eq. (41) have different physical origins-despite their predicted identical dependence on crystal field phase angles within the 6-band quasicubic model. The importance of this distinction will become clear when we consider the effect of long-range exchange in Sec. IV B.

\section{B. Long-range exchange}

In addition to the SR exchange interaction addressed above, the long-range exchange interaction ${ }^{28,42,43}$ also contributes to the fine structure splitting of bound excitons ${ }^{44}$ and in semiconductor NCs. ${ }^{45-50}$ The long-range exchange energy can be calculated directly as the Coulomb energy associated with the exciton polarization. ${ }^{4}$ In the case at hand, considering exciton states $X_{i}=\{X, Y, Z\}$ with orthogonal transition dipoles, we have

$$
\begin{aligned}
H_{X_{i}}^{L R}= & \int_{V_{1}} d V_{1} \int_{V_{2}} d V_{2}\left[-\nabla_{1} \cdot \mathcal{P}_{X_{i}}\left(\boldsymbol{r}_{1}\right)\right]^{*} \frac{1}{\epsilon_{\infty}\left|\boldsymbol{r}_{1}-\boldsymbol{r}_{2}\right|} \\
& \times\left[-\nabla_{2} \cdot \mathcal{P}_{X_{i}}\left(\boldsymbol{r}_{2}\right)\right] .
\end{aligned}
$$

Here, $\epsilon_{\infty}$ is the high frequency dielectric constant, while polarization $\mathcal{P}_{X_{i}}\left(\boldsymbol{r}_{e}\right)$ is the dipole moment density connected with exciton state $X_{i}$. As will be shown later, the LR exchange described by Eq. (44) is proportional to the oscillator strength of the exciton.

Polarization $\mathcal{P}$ is not to be confused with the transition dipole moment in Eq. (39). However, they are related:

$$
\begin{aligned}
\mathcal{P}_{X_{i}}\left(\boldsymbol{r}_{e}\right) & =i \frac{\hbar e}{m_{0} E_{g}} \int d^{3} r_{h} f\left(\boldsymbol{r}_{e}, \boldsymbol{r}_{h}\right) \boldsymbol{p}_{X_{i}} \delta\left(\boldsymbol{r}_{e}-\boldsymbol{r}_{h}\right) \\
& =i \frac{\hbar e}{m_{0} E_{g}} f\left(\boldsymbol{r}_{e}, \boldsymbol{r}_{e}\right) \boldsymbol{p}_{X_{i}} .
\end{aligned}
$$

Here, the unit cell transition momentum matrix element $\boldsymbol{p}_{X_{i}}$ can be rewritten via the dimensionless function $\tilde{p}_{X_{i}}(\theta, \phi)$ that appears in
Eq. (39): $\boldsymbol{p}_{X_{i}}=P \tilde{p}_{X_{i}}(\theta, \phi)$, where $P$ is the Kane matrix element, and we used the relation between matrix elements of the electric dipole and the momentum operators, $\langle 1|\hat{\boldsymbol{\mu}}| 2\rangle=i\left(e \hbar / m_{0}\right)\langle 1|\hat{\boldsymbol{p}}| 2\rangle /\left(E_{2}-E_{1}\right)$, and have approximated the transition energy as $E_{2}-E_{1}=E_{g} / \hbar$. Since we have expressions for the exciton envelope wavefunctions $f\left(\boldsymbol{r}_{e}, \boldsymbol{r}_{h}\right)$, we can thus calculate the polarization and then evaluate the integral over the NC in Eq. (44). Calculations of the exchange splitting created by the LR exchange interaction within the $\boldsymbol{k} \cdot \boldsymbol{P}$ model were conducted in Ref. 19. Accounting for the inequivalence of the reduced energy $\tilde{E}_{X_{i}}$ and the reduced oscillator strength $\tilde{f}_{X_{i}}$ established in Sec. IV A 3, the result can be written in the absence of a dielectric discontinuity at the NC surface as

$$
E_{X_{i}}(\theta, \phi)=\frac{3}{2}\left[\hbar \omega_{S T} \tilde{E}_{X_{i}}(\theta, \phi)+\frac{\hbar \omega_{L T}}{3} \tilde{f}_{X_{i}}(\theta, \phi)\right]\left(\frac{\Theta}{\Theta_{\text {bulk }}}\right) .
$$

In this expression, the size effect term, $\Theta$, is not changed from before, but the magnitude of the splitting is modified by the correction term of magnitude $\hbar \omega_{L T} / 3$, where the bulk longitudinal/transverse (LT) splitting, $\hbar \omega_{L T}$, is given by

$$
\hbar \omega_{L T}=\frac{4}{3 \epsilon_{\infty} a_{x}^{3}} \frac{E_{p}}{m_{0}}\left(\frac{\hbar e}{E_{g}}\right)^{2} .
$$

Here, $E_{p}$ is the Kane energy defined above in Eq. (7). We can thus calculate $\hbar \omega_{L T}$ if we know the band-edge transition dipole, which can be calculated in DFT, or the Kane energy $E_{p}$ can be determined from measured effective masses using, e.g., Eq. (8). For example, the value of $E_{p}$ based on the measured exciton reduced effective mass is given for $\mathrm{CsPbr}_{3}$ in Table I.

\section{Dielectric effects}

One further consideration is that the NC is typically embedded in a medium of dissimilar dielectric constant. In this case, there is an additional correction associated with the interaction of the exciton polarization in Eq. (44) with surface image charges. The leading order correction was estimated in Ref. 19. The resulting correction is parameterized in terms of the ratio, $\kappa=\epsilon_{\infty}^{N C} / \epsilon_{\infty}^{\text {med }}$, of the high frequency dielectric inside the NC, $\epsilon_{\infty}^{N C}$, to that in the surrounding medium, $\epsilon_{\infty}^{\text {med }}$, resulting in the total exchange energy,

$$
\begin{aligned}
E_{X_{i}}(\theta, \phi)= & \frac{3}{2}\left\{\hbar \omega_{S T} \tilde{E}_{X_{i}}(\theta, \phi)+\frac{\hbar \omega_{L T}}{3}\left[1+\frac{12}{\pi^{2}}\left(\frac{\kappa-1}{\kappa+2}\right)\right]\right. \\
& \left.\times \tilde{f}_{X_{i}}(\theta, \phi)\right\}\left(\frac{\Theta}{\Theta_{\text {bulk }}}\right) .
\end{aligned}
$$

\section{Shape effects}

Another important effect which has been shown to affect the exciton fine structure in MHP NCs is NC shape anisotropy. ${ }^{17,1}$ Referring to Eq. (44), it is clear that an asymmetry in the shape of a $\mathrm{NC}$, such as an elongation along one axis, will act to create a splitting between excitons whose transition dipoles are oriented parallel vs perpendicular to the axis of elongation, even in the absence of intrinsic crystal field splitting. The effect could be significant because there is experimental evidence that $\mathrm{CsPbr}_{3} \mathrm{NCs}$ are elongated along the c-axis by $20 \%$. 
To calculate the effect of such a single-axis shape distortion, we evaluate the polarization density of the exciton using Eq. (44). The shape anisotropy enters the analysis via the boundary conditions on the exciton envelope function, $f\left(\boldsymbol{r}_{e}, \boldsymbol{r}_{h}\right)$, which are written in terms of the ground particle in a box wavefunction $\psi_{\mathrm{gr}}(x, y, z)$ as in Eq. (16) in Sec. III; however, to reflect shape distortion from a cubic shape to a right square prism shape, we set the z-dimension of the NC distinct from the x, y dimensions: $\mathcal{L}_{z} \neq \mathcal{L}_{x}=\mathcal{L}_{y}=\mathcal{L}$. The envelope function is then rewritten in terms of the modified ground particle in the box wavefunction appropriate for this geometry as

$$
\psi_{\mathrm{gr}}(\boldsymbol{r})=\left(\frac{8}{\mathcal{L}^{2} \mathcal{L}_{z}}\right)^{1 / 2} \cos (\pi x / \mathcal{L}) \cos (\pi y / \mathcal{L}) \cos \left(\pi z / \mathcal{L}_{z}\right) .
$$

Taking $r=\mathcal{L}_{z} / \mathcal{L}$, as the anisotropy parameter, we preserve NC volume $L^{3}$ by writing $\mathcal{L}_{z}=L r^{2 / 3}$ and $\mathcal{L}_{x}=\mathcal{L}_{y}=L r^{-1 / 3}$. The calculation of the LR exchange interaction was conducted using Eqs. (44) and (45). Using (45) with the exciton envelope wavefunction $f\left(\boldsymbol{r}_{e}, \boldsymbol{r}_{h}\right)$ and substituting into Eq. (44), we find that the derivatives in Eq. (44) act only on the envelope functions. Pulling out the prefactors and using Eq. (47) and $\tilde{f}_{X_{i}}=\left|\tilde{\boldsymbol{p}}_{X_{i}}(\theta, \phi)\right|^{2}$, we find that the LR exchange energy for exciton state $X_{i}$, where $X_{i}=X, Y$, or $Z$, can then be written,

$$
H_{X_{i}}(r, L)=\frac{\hbar \omega_{L T}}{2} \tilde{f}_{X_{i}} \mathcal{A}_{X_{i}}(r, L)\left(\frac{\Theta_{c}(L)}{\Theta_{\text {bulk }}}\right) .
$$

Here, $\Theta_{c}(L)$ is the exchange overlap function for a cube of volume $L^{3}$, and we have defined anisotropy functions $\mathcal{A}_{X_{i}}(L, r)$ as

$$
\mathcal{A}_{X_{i}}(r, L) \equiv \frac{3}{4 \pi} \frac{\Omega I_{X_{i}}(r, L)}{\Theta_{c}(L)},
$$

where $I_{X_{i}}$ denotes exchange integrals for the $X_{i}$ exciton. The integrals $I_{Z}$ and $I_{X}=I_{Y}$ are given explicitly in terms of the exciton envelope function $f\left(r_{e}, r_{h}\right)$ as

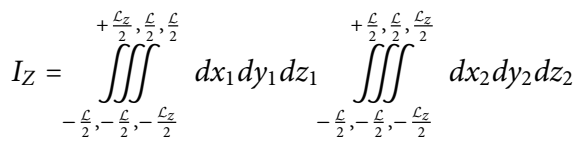

$$
\begin{aligned}
& \times\left[\frac{d f\left(r_{1}, r_{1}\right)}{d z_{1}}\right]^{*} \frac{1}{\left|\boldsymbol{r}_{1}-\boldsymbol{r}_{2}\right|}\left[\frac{d f\left(r_{2}, r_{2}\right)}{d z_{1}}\right], \\
& I_{X}=\int_{-\frac{\mathcal{L}}{2},-\frac{\mathcal{L}}{2},-\frac{\mathcal{L}_{z}}{2}}^{+\frac{\mathcal{L}_{z}}{2}, \frac{\mathcal{L}}{2}, \frac{\mathcal{L}}{2}} d x_{1} d y_{1} d z_{1} \int_{-\frac{\mathcal{L}}{2},-\frac{\mathcal{L}}{2},-\frac{\mathcal{L}_{z}}{2}}^{+\frac{\mathcal{L}}{2}, \frac{\mathcal{L}}{2}, \frac{\mathcal{L}_{z}}{2}} d \int_{2} d y_{2} d z_{2} \\
& \times\left[\frac{d f\left(r_{1}, r_{1}\right)}{d x_{1}}\right]^{*} \frac{1}{\left|\boldsymbol{r}_{1}-\boldsymbol{r}_{2}\right|}\left[\frac{d f\left(r_{2}, r_{2}\right)}{d x_{1}}\right] .
\end{aligned}
$$

Evaluation of Eqs. (51)-(53) in the strong and weak confinement regimes gives identical results and shows that $\mathcal{A}(r)$ is independent of $L$ while $\Theta$ is independent of $r$. In principle, in the intermediate confinement approximation, the normalization factor $N(\beta)$ in Eq. (16) should depend on the anisotropy $r$. This is a small effect, however, for small $|r-1|$, and we neglect it. Additionally, we note that Eq. (50) is derived neglecting image charge effects. Including the dielectric corrections to the lowest order as discussed in Sec. IV B 1 results in a modification of the prefactor in Eq. (50),

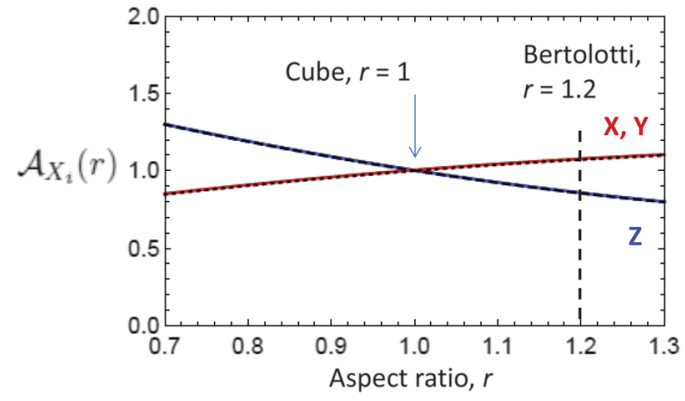

FIG. 5. Effect of NC shape anisotropy on exchange energy. The shape response function, $\mathcal{A}_{X_{i}}$, Eq. (51), is plotted as a function of the aspect ratio $r$ for a uniaxial elongation along the $\mathrm{z}$ direction; here, $r=\mathcal{L}_{z} / \mathcal{L}$, where we preserve NC volume $V=L^{3}$ by writing $\mathcal{L}_{z}=L r^{2 / 3}$ and $\mathcal{L}_{x}=\mathcal{L}_{y}=L r^{-1 / 3}$. The plot shows the relative shift of the $L R$ exchange energy for the $Z$ and orthogonally polarized $X$ and $Y$ excitons due to the shape effect. At a ratio $r=1$, the NC is cube shaped and there is no splitting; for an elongation of $20 \%$ as reported by Bertolotti et al. in Ref. 25 , the $Z$ exciton drops in energy relative to the $X / Y$ doublet by roughly $20 \%$ of the $L R$ exchange energy.

$$
\begin{aligned}
H_{X_{i}}(r, L)= & \frac{3}{2}\left[\frac{\hbar \omega_{L T}}{3}+\hbar \omega_{L T} \frac{4}{\pi^{2}}\left(\frac{\kappa-1}{\kappa+2}\right)\right] \\
& \times \tilde{f}_{X_{i}}(\theta, \phi) \mathcal{A}_{X_{i}}(r, L)\left(\frac{\Theta_{c}(L)}{\Theta_{\text {bulk }}}\right) .
\end{aligned}
$$

Figure 5 shows the calculated dependence of $\mathcal{A}_{X_{i}}$ as a function of the aspect ratio $r$ for a uniaxial elongation along the $\mathrm{z}$ direction; the plot shows the relative shift of the LR exchange energy for the $Z$ and orthogonally polarized $X$ and $Y$ excitons due to the shape effect.

\section{CALCULATED FINE STRUCTURE: $\mathrm{CsPbBr}_{3} \mathrm{NCs}$}

Putting together all of the results derived above, we can now write an expression for the exchange-related fine structure for a MHP NC with intrinsic crystal field splitting and a shape anisotropy, accounting for both short- and long-range exchange. The result for the dark exciton singlet state is just $E_{D}=0$, while for the triplet exciton states $X_{i}=X, Y$, or $Z$, the result is

$$
\begin{aligned}
E_{X_{i}}(\theta)= & \frac{3}{2}\left\{\hbar \omega_{S T} \tilde{E}_{X_{i}}+\left[\frac{\hbar \omega_{L T}}{3}+\hbar \omega_{L T} \frac{4}{\pi^{2}}\left(\frac{\kappa-1}{\kappa+2}\right)\right]\right. \\
& \left.\times \tilde{f}_{X_{i}} \mathcal{A}_{X_{i}}(r, L)\right\}\left(\frac{\Theta(L)}{\Theta_{\text {bulk }}}\right) .
\end{aligned}
$$

We note that within the quasicubic model, the reduced energy $\tilde{E}_{X_{i}}$ and the reduced oscillator strength $\tilde{f}_{X_{i}}$ are equal; however, the DFT calculations presented in Sec. IV A 3 clearly show that these are generally unequal. To factor this into account, we have distinguished between the reduced energy and reduced oscillator strength in the expression for the exchange energy for each exciton state $X_{i}$. Within the context of the quasicubic model, we can then write $\tilde{E}_{X_{i}}=\tilde{E}_{X_{i}}\left(\theta_{E}, \phi_{E}\right)$ and $\tilde{f}_{X_{i}}=\tilde{f}_{X_{i}}\left(\theta_{f}, \phi_{f}\right)$ to reflect the fact that the effective phase angles that determine the reduced energy $\left(\theta_{E}\right.$ and $\left.\phi_{E}\right)$ and reduced oscillator strengths $\left(\theta_{f}\right.$ and $\left.\phi_{f}\right)$ are different, as shown in the DFT calculations performed for MHP structures of tetragonal and orthorhombic symmetry. This is shown in Tables V and VI, 
respectively. The effect of the different phase angles was not taken into account in Ref. 19.

To illustrate the use of these expressions, we apply them to calculate the fine structure for $\mathrm{CsPBr}_{3} \mathrm{NCs}$ with tetragonal, or apparently tetragonal, symmetry, motivated by the experimental studies of Fu et al. ${ }^{12}$ and Ramade et al. ${ }^{14}$ Both of these studies reported single $\mathrm{Cs} \mathrm{PbBr}_{3} \mathrm{NC}$ photoluminescence spectra at liquid He temperature consisting of polarized doublets with a linearly polarized, lower-lying singlet line, which were ascribed to the existence of polyphase NCs with some NCs having tetragonal lattice structure. In the Fu study, magneto-optic measurements on a single NC of $\sim 9 \mathrm{~nm}$ size demonstrated magnetic field splitting of the upper line, consistent with an assignment as an unresolved doublet, as expected for tetragonal symmetry.

Figure 6 shows the size dependence of the exciton fine structure levels in $\mathrm{CsPbBr}$ NCs assumed to have tetragonal lattice structure. In panel (a), we show the fine structure calculated with Eq. (55) including both short-range exchange and long-range exchange, for cube shaped NCs, while in panel (b) we show the calculation for NCs elongated $20 \%$ along the c-axis, taken as the z-direction, consistent with the measurements of Bertolotti et al. ${ }^{25}$ In both cases, the point symmetry is $D_{4 h}$. For these calculations, we used the shortrange exchange constant and the reduced energies $\tilde{E}_{X_{i}}$ and oscillator strengths $\tilde{f}_{X_{i}}$ calculated in DFT for tetragonal $\mathrm{CsPbBr}_{3}$ shown in
Table V. To determine the size dependence, we used the exciton radius, effective mass, and effective dielectric constant from the low temperature measurement of Yang et al., ${ }^{20}$ shown in Table I, and the $\Theta\left(L / a_{x}\right)$ calculated in the intermediate confinement regime, shown in Fig. 4. The corresponding bulk singlet-triplet splitting parameter $\hbar \omega_{s t}=0.7 \mathrm{meV}$. We calculated the fine structure energies using two values for the long-range exchange parameter $\hbar \omega_{L T}$. Solid lines in the figure are calculated with $\hbar \omega_{L T}=5.4 \mathrm{meV}$, as reported in Ref. 21, which matches the value calculated using Eq. (47) using the Kane energy $E_{p}=27.9 \mathrm{eV}$ derived from the experimental effective mass data of Yang et al. and using a high frequency dielectric of $\epsilon_{\infty}=4.8$, consistent with measured refractive index data for $\mathrm{CsPbBr}_{3}{ }^{51} \mathrm{We}$ also show the calculation using a lower value of the Kane energy, $E_{p}=14.3 \mathrm{eV}$, calculated by DFT, corresponding to $\hbar \omega_{L T}=2.8 \mathrm{meV}$. In both cases, the dielectric corrections in Eq. (55) are calculated using $\kappa=2.1$ for a NC with a high frequency dielectric of $\epsilon_{\infty}=4.8$ embedded in a medium of a refractive index of $n=1.49$.

In panel (a) of the figure, calculated for cube-shaped NCs, the bright $Z$ exciton is higher in energy than the $X / Y$ exciton levels. This level order obtains regardless of the value of the Kane energy, or the NC size, since the level order is determined only by the crystal field parameters in this calculation. As noted in Ref. 19, the calculated level order is opposite to the level order reported for apparently
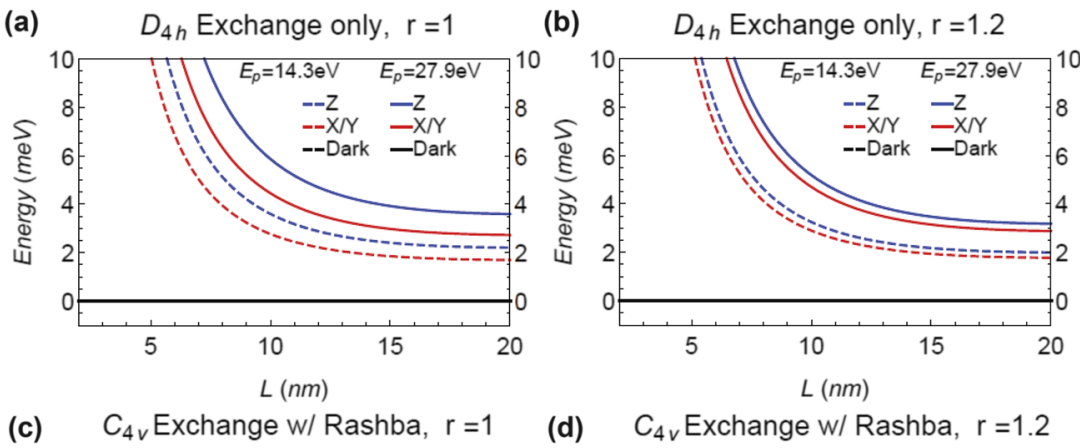

(d) $\quad C_{4 v}$ Exchange $w /$ Rashba, $r=1.2$
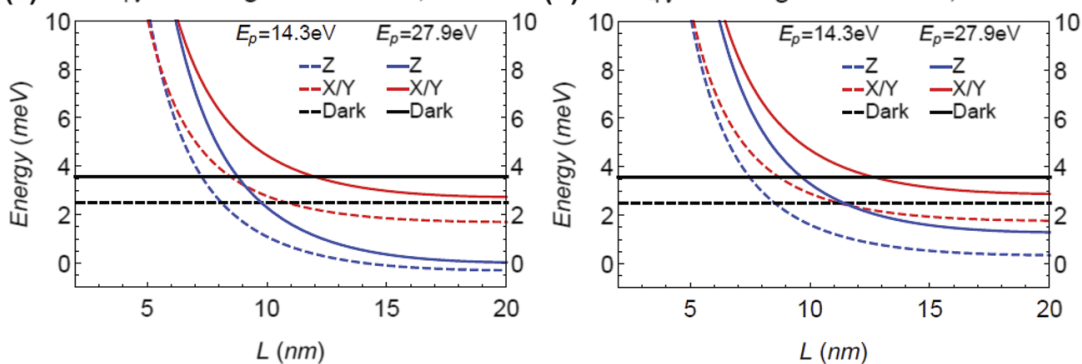

FIG. 6. Exciton fine structure of $\mathrm{CsPbBr}_{3} \mathrm{NCs}$ with tetragonal crystal symmetry vs NC size. Plots show fine structure energy plotted vs edge length $L$ of a cubeshaped NC. The top panels show results accounting for electron-hole exchange only, for a cube-shaped NC $(r=1)$ in panel (a) and for a NC elongated along the $\hat{z}$, or $\mathrm{c}$, direction by $20 \%(r=1.2)$ in panel (b). In both cases, the point symmetry is $D_{4 h}$. The fine structure is calculated using the Kane energy of $E_{p}=27.9$ eV, solid lines, based on the experimental effective mass data from Ref. 20, using the high frequency dielectric of $\epsilon_{\infty}=4.8$ in order to match the bulk LT splitting energy of $\hbar \omega_{L T}=5.4 \mathrm{meV}$ measured in Ref. 21. The resulting $\kappa=2.1$ for a NC embedded in a medium of a refractive index of $n=1.49$. Additionally, we show calculations in dashed lines using $E_{p}=14.3 \mathrm{eV}$, based on the DFT calculation, with all other parameters being the same. In panels (c) and (d), the exciton fine structure is shown with the addition of a phenomenological exciton Rashba energy $\mathcal{E}_{R}$ set for each set of parameters to reproduce the experimental ${ }^{14} 1$ meV splitting between the $Z$ and $X / Y$ excitons at a NC size of $7 \mathrm{~nm}$. All other parameters are the same between panel (c) and panel (a) and between panel (d) and panel (b). The point symmetry for the structures modeled in panels (c) and (d) is $C_{4 v}$, reflecting inversion symmetry breaking along the $z$ axis. 
tetragonal $\mathrm{CsPbBr}_{3} \mathrm{NCs}^{12,14}$ and runs contrary to the theoretical explanation given in Refs. 12 and 14, where the level structure was explained in terms of a negative tetragonal crystal field. Panel (b) in the figure shows the fine structure calculated for NCs elongated by $20 \%$ along the $z$ direction, consistent with recent experimental measurements of the shape of $\mathrm{CsPbBr}_{3} \mathrm{NCs}^{25}$ These calculations also produce a level order with the $Z$ exciton above the $X / Y$ doublet, albeit with a smaller energy splitting between these levels than in the calculations shown in panel (a) for the perfect cubic shape. Notably, this level order is still inconsistent with the measurements of Refs. 12 and 14, despite the significant elongation along the $z$ direction. Although in fact the elongation along the $z$-direction, in conjunction with the LR exchange, tends to reduce the $Z$ exciton energy and increase the energy of the $X / Y$ doublet as shown in Fig. 5, this effect is insufficient to overcome the effect of the tetragonal crystal field. In particular, reference to the DFT calculation for the tetragonal $\mathrm{CsPbBr}_{3}$ structure in Table $\mathrm{V}$ shows that there is a significantly larger oscillator strength for the $Z$ exciton, $\tilde{f}_{Z}$, than for the $X / Y$ exciton, $\tilde{f}_{X / Y}$, which directly affects the LR exchange contribution to the energy and pushes the $Z$ exciton upwards [see Eq. (55)].

In order to describe the observed level order, we retrace the argument made in Becker et al., ${ }^{16}$ pertaining to the effect of Rashba terms on the exciton fine structure. In that paper, it was proposed that inversion breaking in conjunction with the large spin-orbit coupling in $\mathrm{CsPbX}_{3}$ would create Rashba terms which were shown to lead to corrections to the exciton fine structure energies. For a NC of tetragonal symmetry with inversion symmetry breaking along the $z$ axis, the correction is given by ${ }^{16}$

$$
\Delta E^{R}=-2\left(A_{c}-A_{r}\right) \mathcal{E}_{R}\left(\tau_{x}^{e} \tau_{x}^{h}+\tau_{y}^{e} \tau_{y}^{h}\right) .
$$

Here, $A_{c}=128 /\left(27 \pi^{2}\right) \sim 0.48$ and $A_{r}=(64 / 81 \sqrt{3})^{2} \sim 0.21$ are numerical coefficients reflecting the center-of-mass and relative motion of the exciton, and $\mathcal{E}_{R}=\alpha_{e} \alpha_{h} \mu / \hbar^{2}$ is the exciton Rashba energy, given in terms of Rashba coefficients of the electron and hole ( $\alpha_{e}$ and $\alpha_{h}$, respectively) and the exciton reduced effective mass $\mu$. Most importantly, the operators $\tau_{i}$ in this expression are the projections of the Pauli operators for the electron total angular momentum operator for $J=1 / 2$ and for the hole spin $s=1 / 2$, ( $\tau_{i}^{e}$ and $\tau_{i}^{h}$, respectively). [We denote these operators as $\tau_{i}$ to distinguish them from the true spin Pauli operators $\sigma_{i}$ used in Eq. (24).] For Rashba coefficients $\alpha_{e}$ and $\alpha_{h}$ with the same sign, the effect of this term is to create energy shifts opposite to that of the electron-hole exchange interaction. In the tetragonal case specifically, it tends toward a reversal of the order of levels between the $Z$ exciton and the dark singlet, without affecting the energy position of the $X Y$ doublet. The $Z$ state moves down in energy by $-4\left(A_{c}-A_{r}\right) \mathcal{E}_{R} \sim-1.09 \mathcal{E}_{R}$, while the dark state moves upwards by $+4\left(A_{c}-A_{r}\right) \mathcal{E}_{R} \sim 1.09 \mathcal{E}_{R}$.

In panels (c) and (d) of Fig. 6, we show the effect of the Rashba term on the size-dependent exciton fine structure for a cube-shaped and an elongated NC, respectively. For both these panels, the point symmetry of the structure is $C_{4 v}$ reflecting inversion symmetry breaking along the $z$ direction. These calculations include exchange, using exchange and shape parameters exactly as in panels (a) and (b) but with the addition of a phenomenological Rashba correction, Eq. (56), with exciton Rashba energy $\mathcal{E}_{R}$ fixed for each set of parameters to reproduce the experimental ${ }^{14} 1 \mathrm{meV}$ splitting between the $Z$ and $X / Y$ excitons at a NC size of $7 \mathrm{~nm}$. All other parameters are the same between panel (c) and panel (a) and between panel (d) and panel (b). This requires values $\mathcal{E}_{R}=2.30,3.27 \mathrm{meV}$ in panel (c) and $\mathcal{E}_{R}=1.51,1.73 \mathrm{meV}$ in panel (d), for values of $E_{p}=14.2$ and $27.9 \mathrm{eV}$, respectively. These values correspond to Rashba coefficients ranging from $0.30 \mathrm{eV}$ - $\AA$ to $0.45 \mathrm{eV}-\AA$, assuming that the electron and hole coefficients are equal.

From the plots, it is clear that the bright state level order in both panels (c) and (d) agrees with the experimental level order reported by $\mathrm{Fu}$ et $a l .{ }^{12}$ and Ramade et al. ${ }^{14}$ Importantly, the model predicts that for NCs larger than $\sim-10 \mathrm{~nm}$ depending on the parameters, the ground exciton state is a bright state, as proposed in Ref. 16. However, the model indicates that this will not be true in small NCs where the exchange splitting, which increases as $\sim 1 / L^{3}$ with decreasing size $L$, exceeds the Rashba term. For small NCs, the ground state must be a dark exciton state.

An important point that becomes apparent upon inspection of panels (c) and (d) of Fig. 6 is that, when the Rashba term is present, the bright state level spacing decreases with decreasing size of the NCs, which is opposite from the behavior expected in a pure exchange model. In the latter case, the fine structure splitting increases with decreasing size $L$ as $\sim 1 / L^{3}$, as shown in panels

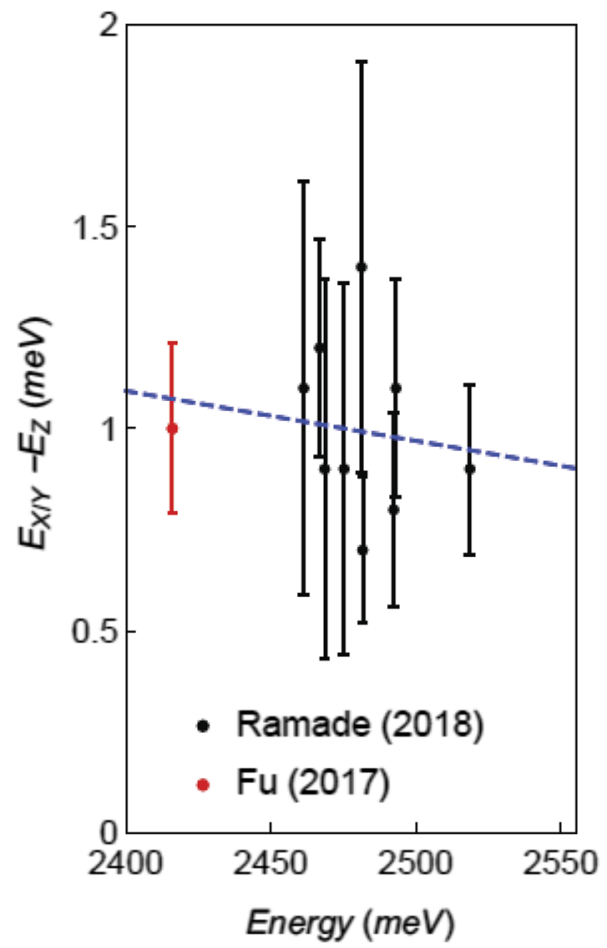

FIG. 7. Energy of fine structure splitting in tetragonal $\mathrm{CsPbBr}_{3} \mathrm{NCs}$. Data are taken from the work of Ramade et al..$^{14}$ and Fu et al. ${ }^{12}$ The dashed line shows the average dependence of the splitting as a function of emission energy, calculated via a best linear fit. Error bars are estimated as $1 \sigma$ values assuming Gaussian line shapes. 
(a) and (b) of the same figure. The size dependence of the splitting between the optically active states is thus an important feature that can be tested experimentally to distinguish whether the fine structure is determined purely by exchange or whether the Rashba effect plays an important role. Significantly, this assessment can be made without direct detection of the dark exciton state. To illustrate this type of analysis with the available experimental data, Fig. 7 shows the measured fine structure splitting in nominally tetragonal $\mathrm{Cs} \mathrm{PbBr}_{3}$ NCs reported in Refs. 12-14. The plot shows that the fine structure splitting is not increasing with increasing exciton emission energy as would be expected in a pure exchange model; indeed, a best line fit through the data indicates a slight decrease in splitting with increasing emission energy, as noted in Ref. 14. While this is certainly not a definitive test given the variability in the data, the result is suggestive and should motivate further studies of the size-dependent fine structure splitting over a broader range of NC sizes.

\section{DISCUSSION OF FINE STRUCTURE IN OTHER MHP NCS}

Recently, magnetic activation of the lower, dark ground singlet level predicted by the exchange model was directly detected in single $\mathrm{FAPbBr}_{3} \mathrm{NCs}$ by Tamarat et al. ${ }^{15}$ This result shows that Rashba terms which can reverse the singlet/triplet level order in MHP systems ${ }^{16}$ must not exist in this material. Additionally, the fine structure splitting was in fact observed to increase with increasing exciton emission energy as expected for a fine structure dominated by the electron-hole exchange interaction. Referring to the discussion in Sec. $V$, this result is in contrast to what has been reported so far ${ }^{12,14}$ for $\mathrm{CsPbr}_{3} \mathrm{NCs}$ where magnetic detection of a lower dark singlet has not been observed.

Regarding the specifics of the exchange interaction in $\mathrm{FAPbBr}_{3}$ $\mathrm{NCs}$, our results here indicate that the interplay between the intrinsic crystal field and NC shape asymmetry is expected to work out differently for $\mathrm{FAPbBr}_{3}$ than in $\mathrm{CsPbBr}_{3}$ NCs. Referring to Table V, for the calculations done here on the $\mathrm{FAPbBr}_{3}$ mimic structure, the crystal field is very small so that the SR exchange splitting and the difference in the oscillator strengths of the $\mathrm{X}, \mathrm{Y}$, and $\mathrm{Z}$ excitons are found to be almost negligible, as assumed in the theoretical analysis of Ref. 15. As a result, the shape effect should dominate over the intrinsic crystal field in this system, leading to a level structure with the singlet $Z$ state below the $X / Y$ doublet, as observed in Ref. 15. Along the same lines, reviewing the DFT calculations for the orthorhombic $\mathrm{FAPbBr}_{3}$ mimic structure in Table VI, the order of the reduced energies $\tilde{E}_{X_{i}}$ and the reduced oscillator strengths $\tilde{f}_{X_{i}}$ are opposite each other. Equivalently, the crystal field $\delta$ determined from the SR exchange energies vs the oscillator strengths have opposite signs. Thus, the SR exchange and LR exchange terms tend to cancel each other, leading again to minimal net crystal field splitting.

\section{SUMMARY}

To summarize, a general description of the size dependence of the lowest electron, hole, and exciton levels in spherical and cube-shaped NCs of metal halide perovskites was developed within simple parabolic and nonparabolic coupled-band effective-mass models. The semianalytical expressions derived in this paper accurately reproduce results of the exact theoretical solutions, which in turn provide an excellent description of the experimental size dependence of the exciton absorption line in cube-shaped $\mathrm{Cs} \mathrm{PbBr}_{3} \mathrm{NCs}$. We also presented a complete description of the size-dependent exciton fine structure in cube-shaped and nearly cube-shaped metal halide perovskite NCs that takes into account the short- and longrange exchange interactions; cubic, tetragonal, and orthorhombic crystal structure; the effect of dielectric confinement; and the contribution of the Rashba effect; as well as the effect of elongation of the NC shape. The analytical theoretical analysis of the exciton fine structure that was conducted within the quasicubic 6-band model was assessed against first principles hybrid DFT calculations. The comparison of the two approaches provides an important insight into the relationship between the oscillator transition strengths and the exchange splitting of the three bright excitons, which is oversimplified within a 6 band effective mass model. DFT calculations conducted here provide parameters necessary for the description of the exciton fine structure in $\mathrm{CsPbBr}_{3}, \mathrm{FAPbBr}_{3}$, and $\mathrm{MAPbI}_{3}$ perovskites. The comparison of our theoretical calculations of the exciton fine structure with PL spectra measured in single $\mathrm{Cs} \mathrm{PbBr}_{3}$ NCs of different sizes and the absence of the dark exciton line in the reported spectra suggest that an important role is played by the Rashba effect in these NCs.

\section{SUPPLEMENTARY MATERIAL}

See the Supplementary material for the coupled band $\boldsymbol{k} \cdot \boldsymbol{P}$ model for quantum confined carrier states in spherical NCs, derivation and analysis of the validity of the quasicubic model for orthorhombic lattice symmetry, and details of the DFT band structure and exchange calculations.

\section{ACKNOWLEDGMENTS}

P.C.S. acknowledges support from the Center for Hybrid Organic Inorganic Semiconductors for Energy (CHOISE), an Energy Frontier Research Center funded by the Office of Basic Energy Sciences, Office of Science within the U.S. Department of Energy. J.L.L., N.B., and A.L.E. acknowledge support from the U.S. Office of Naval Research. The work of J.L.L., and A.L.E was supported by the Laboratory University Collaboration Initiative of the DoD Basic Research Office.

The authors declare no competing financial interests.

\section{REFERENCES}

${ }^{1}$ L. Protesescu, S. Yakunin, M. I. Bodnarchuk, F. Krieg, R. Caputo, C. H. Hendon, R. X. Yang, A. Walsh, and M. V. Kovalenko, Nano Lett. 15, 3692 (2015).

${ }^{2}$ G. Rainò, G. Nedelcu, L. Protesescu, M. I. Bodnarchuk, M. V. Kovalenko, R. F. Mahrt, and T. Stöferle, ACS Nano 10, 2485 (2016).

${ }^{3}$ S. A. Crooker, T. Barrick, J. A. Hollingsworth, and V. I. Klimov, Appl. Phys. Lett. 82, 2793 (2003)

${ }^{4}$ L. Biadala, B. Siebers, Y. Beyazit, M. D. Tessier, D. Dupont, Z. Hens, D. R. Yakovlev, and M. Bayer, ACS Nano 10, 3356 (2016).

${ }^{\mathbf{5}}$ B. T. Diroll, H. Zhou, and R. D. Schaller, Adv. Funct. Mater. 28, 1800945 (2018).

${ }^{6}$ M. D. Nirmal, J. Norris, M. Kuno, M. G. Bawendi, Al. L. Efros, and M. Rosen, Phys. Rev. Lett. 75, 3728 (1995). 
${ }^{7}$ Al. L. Efros, M. Rosen, M. Kuno, M. Nirmal, D. J. Norris, and M. G. Bawendi, Phys. Rev. B 54, 4843 (1996).

${ }^{8}$ M. Certier, C. Wecker, and S. Nikitine, J. Phys. Chem. Solids 30, 2135 (1969).

${ }^{9}$ D. Canneson, E. V. Shornikova, D. R. Yakovlev, T. Rogge, A. A. Mitioglu, M. V. Ballottin, P. C. M. Christianen, E. Lhuillier, M. Bayer, and L. Biadala, Nano Lett. 17, 6177 (2017).

${ }^{10}$ L. Chen, B. Li, C. Zhang, X. Huang, X. Wang, and M. Xiao, Nano Lett. 18, 2074 (2017).

${ }^{11}$ K. Xu, J. F. Vliem, and A. Meijerink, J. Phys. Chem. C 123, 979 (2019).

${ }^{12} \mathrm{M}$. Fu, P. Tamarat, H. Huang, J. Even, A. L. Rogach, and B. Lounis, Nano Lett. 17, 2895 (2017).

${ }^{13}$ M. Isarov, L. Z. Tan, M. I. Bodnarchuk, M. V. Kovalenko, A. M. Rappe, and E. Lifshitz, Nano Lett. 17, 5020 (2017).

${ }^{14}$ J. Ramade, L. M. Andriambariarijaona, V. Steinmetz, N. Goubet, L. Legrand, T. Barisien, F. Bernardot, C. Testelin, E. Lhuillier, A. Bramati, and M. Chamarro, Nanoscale 10, 6393 (2018)

${ }^{15}$ P. Tamarat, M. I. Bodnarchuk, J.-B. Trebbia, R. Erni, M. V. Kovalenko, J. Even, and B. Lounis, Nat. Mater. 18, 717 (2019).

${ }^{16}$ M. A. Becker, R. Vaxenburg, G. Nedelcu, P. C. Sercel, A. Shabaev, M. J. Mehl, J. G. Michopoulos, S. G. Lambrakos, N. Bernstein, J. L. Lyons, T. Stöferle, R. F. Mahrt, M. V. Kovalenko, D. J. Norris, G. Raino, and Al. L. Efros, Nature 553, 189 (2018).

${ }^{17}$ M. O. Nestoklon, S. V. Goupalov, R. I. Dzhioev, O. S. Ken, V. L. Korenev, Yu. G. Kusrayev, V. F. Sapega, C. de Weerd, L. Gomez, T. Gregorkiewicz, J. Lin, K. Suenaga, Y. Fujiwara, L. B. Matyushkin, and I. N. Yassievich, Phys. Rev. B 97, 235304 (2018).

${ }^{18}$ R. Ben Aich, I. Saidi, S. Ben Radhia, K. Boujdaria, T. Barisien, L. Legrand, F. Bernardot, M. Chamarro, and C. Testelin, Phys. Rev. Appl. 11, 034042 (2019).

${ }^{19}$ P. C. Sercel, J. L. Lyons, D. Wickramaratne, R. Vaxenburg, N. Bernstein, and Al. L. Efros, Nano Lett. 19, 4068 (2019).

${ }^{20}$ Z. Yang, A. Surrente, K. Galkowski, A. Miyata, O. Portugall, R. J. Sutton, A. A. Haghighirad, H. J. Snaith, D. K. Maude, P. Plochocka, and R. J. Nicholas, ACS Energy Lett. 2, 1621 (2017).

${ }^{21}$ V. V. Belykh, D. R. Yakovlev, M. M. Glazov, P. S. Grigoryev, M. Hussain, J. Rautert, D. N. Dirin, M. V. Kovalenko, and M. Bayer, Nat. Commun. 10, 673 (2019).

${ }^{22}$ I. Kang and F. W. Wise, J. Opt. Soc. Am. B 14, 1632 (1997).

${ }^{23}$ M. C. Brennan, J. E. Herr, T. S. Nguyen-Beck, J. Zinna, S. Draguta, S. Rouvimov, J. Parkhill, and M. Kuno, J. Am. Chem. Soc. 139, 12201 (2017).

${ }^{24}$ J. Even, L. Pedesseau, J.-M. Jancu, and C. Katan, J. Phys. Chem. Lett. 4, 2999 (2013).

${ }^{25}$ F. Bertolotti, L. Protesescu, M. V. Kovalenko, S. Yakunin, A. Cervellino, S. J. L. Billinge, M. W. Terban, J. S. Pedersen, N. Masciocchi, and A. Guagliardi, ACS Nano 11, 3819 (2017).

${ }^{26}$ In the same manner, NC shape distortions consisting of elongation along one axis, as in Ref. 25, correspond to spherical harmonics with $L=2$ and can therefore have no effect in first order. This is unlike the situation in semiconductors such as CdSe with orbitally degenerate valence bands where ellipsoidal distortions can affect the quantum size levels in first order, see, e.g., Al. L. Efros and A. V. Rodina, Phys. Rev. B. 47, 10005 (1993).

${ }^{27}$ Y. Wang, A. Suna, W. Mahler, and R. Kasowski, J. Chem. Phys. 87, 7315 (1987).

${ }^{28}$ U. Rossler and H.-R. Trebin, Phys. Rev. B 23, 1961 (1981).

${ }^{29}$ G. L. Bir and G. E. Pikus, Symmetry and Strain-Induced Effects in Semiconductors (Wiley, 1974).

${ }^{30}$ J. Even, L. Pedesseau, C. Katan, M. Kepenekian, J.-S. Lauret, D. Sapori, and E. Deleport, J. Phys. Chem. C 119, 10161 (2015).

${ }^{31}$ Y. Nagamune, S. Takeyama, and N. Miura, Phys. Rev. B 43, 12401 (1991).

${ }^{32}$ Z. G. Yu, Sci. Rep. 6, 28576 (2016).

${ }^{33}$ C. C. Stoumpos, C. D. Malliakas, J. A. Peters, Z. Liu, M. Sebastian, J. Im, T. C. Chasapis, A. C. Wibowo, D. Y. Chung, A. J. Freeman, B. W. Wessels, and M. G. Kanatzidis, Cryst. Growth Des. 13, 2722 (2013).

${ }^{34}$ S. Sun, "Synthesis, characterization and properties of hybrid organic-inorganic perovskites for photovoltaic applications," Ph.D. thesis, University of Cambridge, 2017.

${ }^{35}$ K. Frohna, T. Deshpande, J. Harter, W. Peng, B. A. Barker, J. B. Neaton, S. G. Louie, O. M. Bakr, D. Hsieh, and M. Bernardi, Nat. Commun, 9, 1829 (2018).

${ }^{36}$ E. C. Schueller, G. Laurita, D. H. Fabini, C. C. Stoumpos, M. G. Kanatzidis, and R. Seshadri, Inorg. Chem. 57, 695 (2018).

${ }^{37}$ K. Persson, Materials Data on H6PbCI3N (SG:62) by Materials Project, 2016, https://doi.org/10.17188/1317032.

${ }^{38}$ W. Kohn and L. J. Sham, Phys. Rev. 140, A1133 (1965).

${ }^{39}$ J. Heyd, G. E. Scuseria, and M. Ernzerhof, J. Chem. Phys. 118, 8207 (2003).

${ }^{40}$ G. Kresse and J. Furthmüller, Phys. Rev. B 54, 11169 (1996).

${ }^{41}$ I. P. Pashuk, N. S. Pidzyrailo, and M. G. Matsko, Sov. Phys. Sol. State 23, 1263 (1981).

${ }^{42}$ G. E. Pikus and G. L. Bir, Zh. Eksp. Teor. Fiz. 60, 195 (1971) [Sov. Phys. JETP 33, 108 (1971)].

${ }^{43}$ K. Cho, J. Phys. Soc. Jpn. 68, 683 (1999).

${ }^{44}$ G. E. Pikus and G. L. Bir, Zh. Eksp. Teor. Fiz. 62, 324 (1972) [Sov. Phys. JETP 35, 174 (1972)].

${ }^{45}$ S. V. Goupalov and E. L. Ivchenko, J. Cryst. Growth 184-185, 393-397 (1998); Acta Phys. Pol. A 94, 341 (1998).

${ }^{46}$ S. V. Goupalov and E. L. Ivchenko, Fiz. Tverd. Tela 42, 1976 (2000) [Phys. Solid State 42, 2030 (2000)].

${ }^{47}$ H. Ajiki and K. Cho, Phys. Rev. B 62, 7402 (2000).

${ }^{48}$ S. V. Goupalov and E. L. Ivchenko, Fiz. Tverd. Tela 43, 1791-1798 (2001) [Phys. Solid State 43, 1867 (2001)].

${ }^{49}$ H. Ajiki and K. Cho, Int. J. Mod. Phys. 15, 3745 (2001).

${ }^{50}$ P. C. Sercel and Al. L. Efros, Nano Lett. 18, 4061 (2018).

${ }^{51}$ D. N. Dirin, I. Cherniukh, S. Yakunin, Y. Shynkarenko, and M. V. Kovalenko, Chem. Mater. 28, 8470 (2016). 